\title{
A review of the genus Microprosopa Becker, 1894 (Diptera: Scathophagidae) of Russia
}

\author{
Обзор видов рода Microprosopa Becker, 1894 \\ (Diptera: Scathophagidae) фауны России
}

\begin{abstract}
A.L. Ozerov
А. $\Lambda$. Озеров

Zoological Museum, Lomonosov Moscow State University, Bol'shaya Nikitskaya 2, Moscow 125009, Russia.E-mail: ozerov2455@rambler.ru Зоологический музей, Московский государственный университет им. М.В. Ломоносова, Большая Никитская ул., 2, Москва 125009 , Россия.
\end{abstract}

KEY WORDS: Diptera, Scathophagidae, Microprosopa Becker, Russia, review.

КЛЮЧЕВЫЕ СЛОВА: Diptera, Scathophagidae, Microprosopa Becker, Россия, обзор.

ABSTRACT. Flies of the genus Microprosopa Becker, 1894 of Russia, which includes 12 species, are reviewed. Generic and species descriptions and keys for determination of species are given, and data on distributions are summarized. One species, Microprosopa taimyrica sp.n., is described as new to science. Lectotype is designated for Cordylura melanura Zetterstedt, 1838. Three new synonymies are proposed: $M i-$ croprosopa Becker, 1894 = Allomyella Malloch, 1923, syn.n., Microprosopa pallidicauda (Zetterstedt, 1838) = Cordylura melanura Zetterstedt, 1838, syn.n., and Microprosopa unguiculata (Malloch, 1919) = Allomyella borealis Curran, 1927, syn.n.

РЕЗЮМЕ. Дан обзор двукрылых рода Microprosopa Becker, 1894 фауны России, включающего 12 видов. Приведены диагноз рода, описания видов и ключ для определения видов рода Microprosopa России, а также суммированы данные по распространению видов рода на территории России. Описан один новый для науки вид, Microprosopa taimyrica sp.n. Обозначен лектотип для Cordylura melanura Zetterstedt, 1838. Установлены 3 новых синонима: Microprosopa Becker, 1894 = Allomyella Malloch, 1923, syn.n., Microprosopa pallidicauda (Zetterstedt, 1838) = Cordylura melanura Zetterstedt, 1838, syn.n., и Microprosopa unguiculata $($ Malloch, 1919) $=$ Allomyella borealis Curran, 1927, syn.n.

\section{Introduction}

Microprosopa Becker, 1894 is no large genus within the family Scathophagidae. The species from the genus Allomyella Malloch, 1923 are included in the genus Microprosopa in this work also, and Allomyella is considered as subgenus of Microprosopa. The division of species in the genera Microprosopa and Allomyella is based on the absence (Microprosopa) or pre- sence (Allomyella) of one or several hairs on anepimeron. However this character varies in some species of Allomyella [Vockeroth, 1987]: for example, hairs may be present or absent in A. unguiculata (Malloch, 1919) or A. frigida (Holmgren, 1883). The other character to distinguish Microprosopa and Allomyella species is difference in structure of male sternite 5. Microprosopa spp. has sternite 5 symmetrical, with two simple lobes (Figs 28-33), while sternite 5 of Allomyella spp. has bifurcate or trifurcate lobes, symmetrical or asymmetrical (Figs 23-27, 34-38). Molecular analysis based on DNA sequences for Microprosopa or Allomyella has not been done yet.

Genus Microprosopa consists of 24 species distributed in the Northern Hemisphere (6 Nearctic, 11 Palaearctic, 7 Holarctic) [Vockeroth, 1965, 1987; Gorodkov, 1986; Šifner, 2008, 2011, 2013; Ozerov, 2009; Ozerov \& Krivosheina, 2013]. Fauna of Russia included 11 species [Gorodkov, 1986; Humala \& Polevoi, 2009; Ozerov, 2009; Ozerov \& Barkalov, 2014; Ozerov \& Krivosheina, 2013, 2014], but no general works were fulfilled.

Very little is known about the biology of Microprosopa. Species of this genus are usually collected in marshy plots, at banks of lakes covered with Carex spp., in grass along spring banks. Microprosopa lineata (Zetterstedt, 1838 ) is often present on young branches of Salix spp. None of descriptions of larvae is published.

\section{Material and methods}

The majority of specimens examined for this study are deposited in the Zoological Museum, Moscow State University, Russia (ZMUM) and Zoological Institute of Russian Academy of Sciences, St. Petersburg, Russia (ZISP). I also studied some material from the Institute of Systematics and Ecology of Animals, Russian Academy of Sciences, Siberian Branch, Novosibirsk, Russia (ISEA), Canadian National Collection of In- 

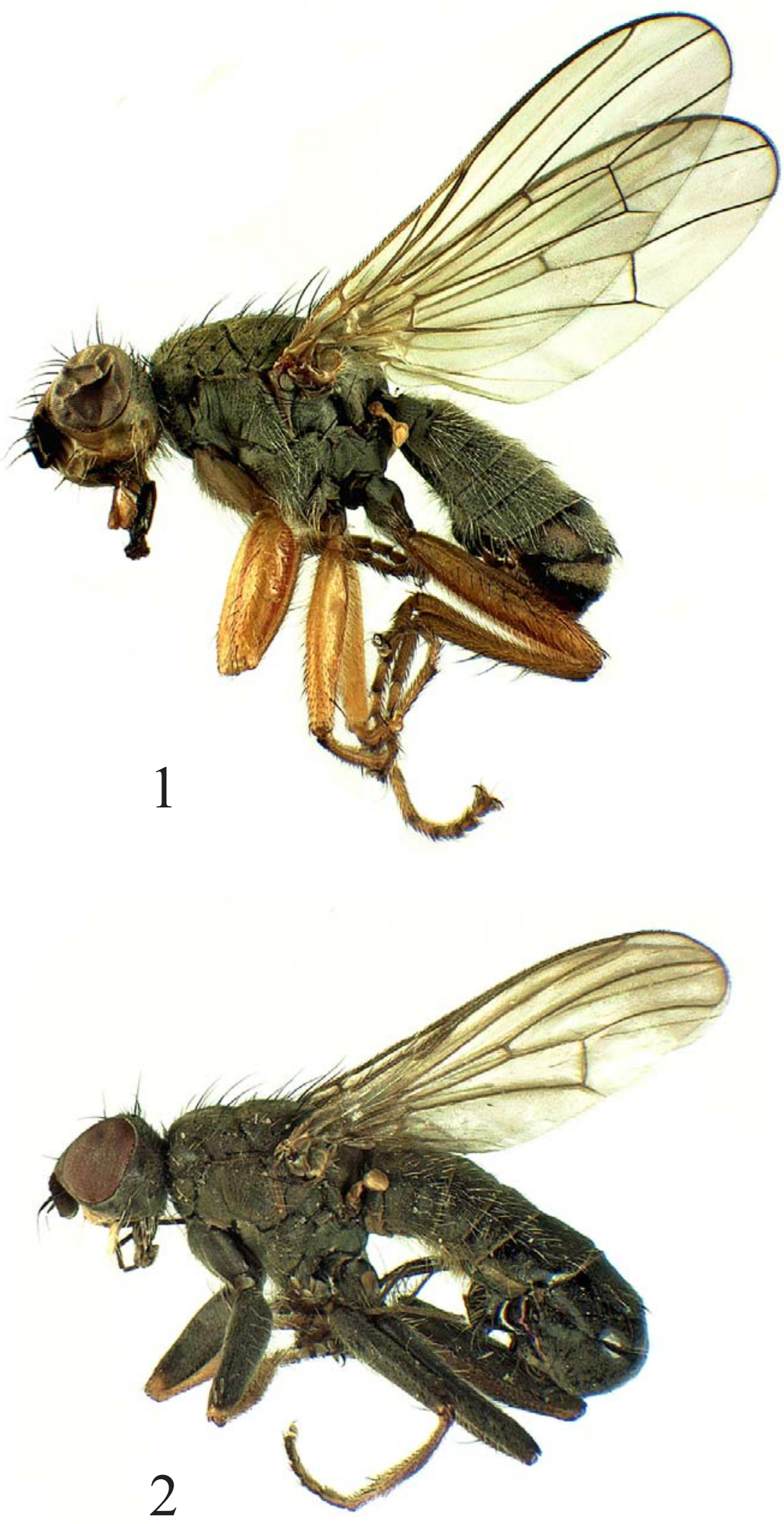

Figs 1-2. Microprosopa pallidicauda (Zetterstedt) (1) and Microprosopa frigida (Holmgren) (2), male habitus, lateral view. Рис. 1-2. Microprosopa pallidicauda (Zetterstedt) (1) и Microprosopa frigida (Holmgren) (2), самец, сбоку. 
sects, Ottawa, Canada (CNC), Finnish Museum of Natural History, Helsinki, Finland (MZH), and Lund University, Lund, Sweden (MZLU).

The majority of original Russian geographical names are given in transliteration, but names of some large geographical regions (e.g. Amur Oblast, Sakhalin Oblast, Khabarovsk Kray, Primorskiy Kray) follows Merriam Webster's Geographical Dictionary [1997].

The data on distributions are cited as follows: Russia is in the first place, other countries of the world are given after an em dash [-]. More detailed data on the distributions of species in Europe may be found in the work by Šifner [2008], and in North America in the work by Vockeroth [1965].

The terminology used in the generic descriptions, species diagnoses, description of the new species, and keys follows McAlpine [1981], Cumming et al. [2009], and Stuckenberg [1999].

Dissected male genitalia were examined with a Nikon SMZ645 zoom stereomicroscope and then photographed using an eTREK DCM900 digital camera attached in place of an eyepiece of monocular microscope. Resulting batches of images were processed with CombineZP software [Hadley, 2007], editing of stacked images was performed in Adobe Photoshop.

\section{Taxonomic part}

\section{Microprosopa Becker, 1894}

Subgenus Microprosopa Becker, 1894: 147. Gender: feminine. Type-species: Cordylura haemorrhoidalis Meigen, 1826, by original designation.

Subgenus Allomyella Malloch, 1923: 199. Gender: feminine. Replacement name for Allomyia Malloch, 1919. Type-species: Allomyia unguiculata Malloch, 1919, automatic - syn.n.

Allomyia Malloch, 1919: 80. Gender: feminine. Junior homonym, preoccupied by Allomyia Banks, 1916. Type-species: Allomyia unguiculata Malloch, by monotypy.

Microprosopa species are small to medium-sized flies (3-10 mm long) (Figs 1, 2).

Head. Frontal vitta from reddish-yellow completely to almost completely black; fronto-orbital plate greyish dusted. Face, parafacial and gena yellow or black. Postcranium black, greyish dusted, covered with black setae in upper third and white hairs in lower part. 3 orbitals, 2-4 frontals, 1 ocellar, 1 small weakly divergent postocellar, 1 inner vertical, 1 outer vertical; $1-2$ pairs of strong vibrissae present. Antenna from yellow to black. Postpedicel with slightly acute upper apical corner or rounded apically, about 2 times as long as wide. Arista black, bare. Palpus broadened towards apex, yellow.

Thorax black, greyish dusted, rare scutum with shining stripes and spots (M. lineta, Fig. 3). Acrostichals as hairs in two rows, dorsocentrals (3-5)+3, including a pair of erect setae on anterior margin of scutum, intra-alars $1+(1-2)$, supra-alars $1+2$, postpronotals 2, notopleurals 2, postalars 2. Proepisternum with hairs in central part, with 1-2 setae near lower margin. Proepimeron with 1-2 setae or setulae, sometimes with

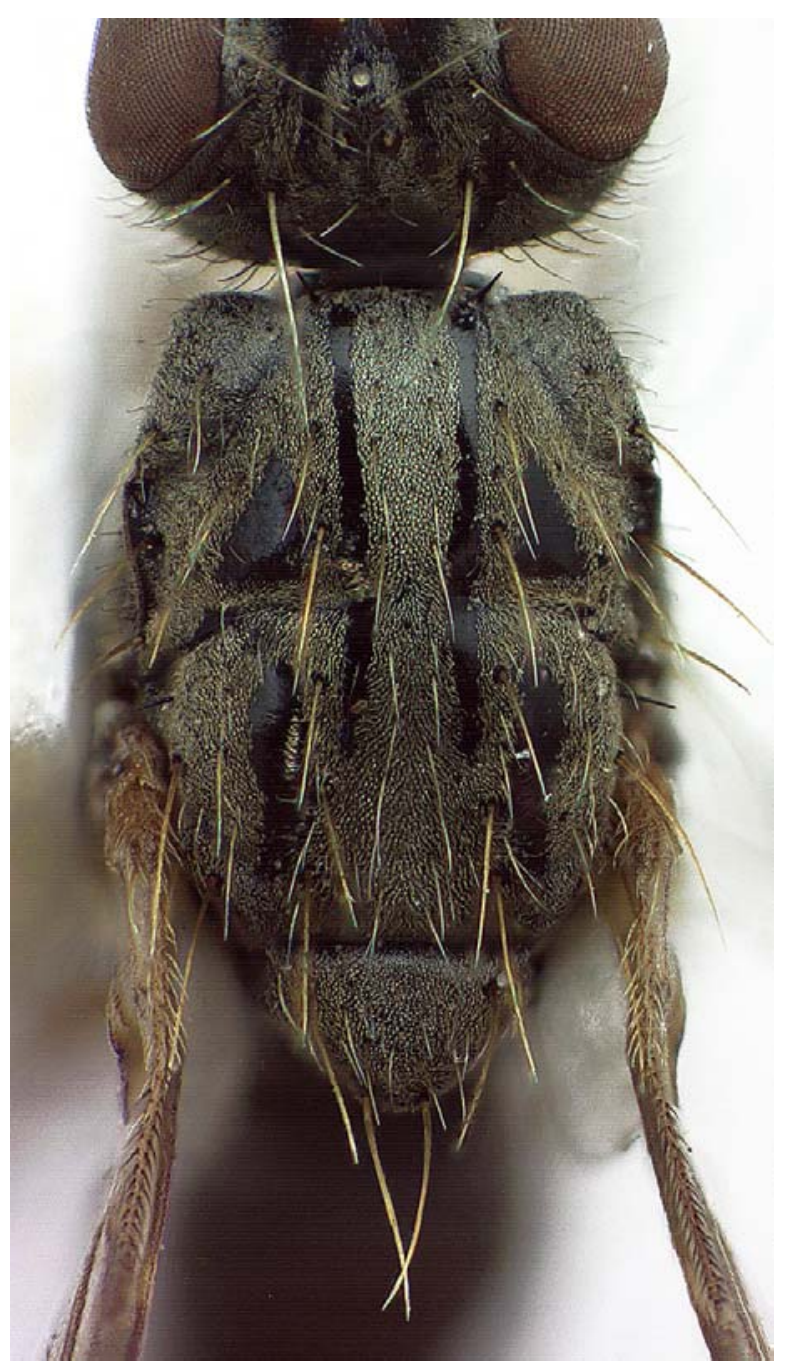

Fig 3. Microprosopa lineata (Zetterstedt), scutum.

Рис. 3. Microprosopa lineata (Zetterstedt), среднеспинка.

a few hairs only. Anepisternum covered with hairs in posterior half, with 1-4 black setae along posterior margin. Katepisternum covered with pale hairs in posterior half and 1 strong seta in upper posterior corner. Anepimeron bare or with a few hairs. Postmetacoxal bridge absent. Scutellum black, greyish dusted, with a pair of basal scutellar and a pair of apical scutellar setae.

Legs. Coxae and fore femora yellow to black, tibiae usually always yellow, tarsi yellow. Fore femur simple, without numerous black short spinules ventrally (Fig. 4). Fore tibia with short black spinules ventrally, usually in two rows on whole length (Fig. 4), with 1 dorsal seta near middle, 1 preapical dorsal, $0-1$ apical anterodorsal and usually with 1 apical posterior setae. Mid femur with irregular row of anterior setae, with 1 preapical posterior and 1 preapical posterodorsal setae. Mid tibia with 1 anterodorsal and $0-1$ posterior setae near middle, and a ring of apicals. Hind femur with a row of anterodorsal setae. Hind tibia with 2-3 anterodorsal, 1-2 posterodorsal and 1 preapical dorsal setae, also 

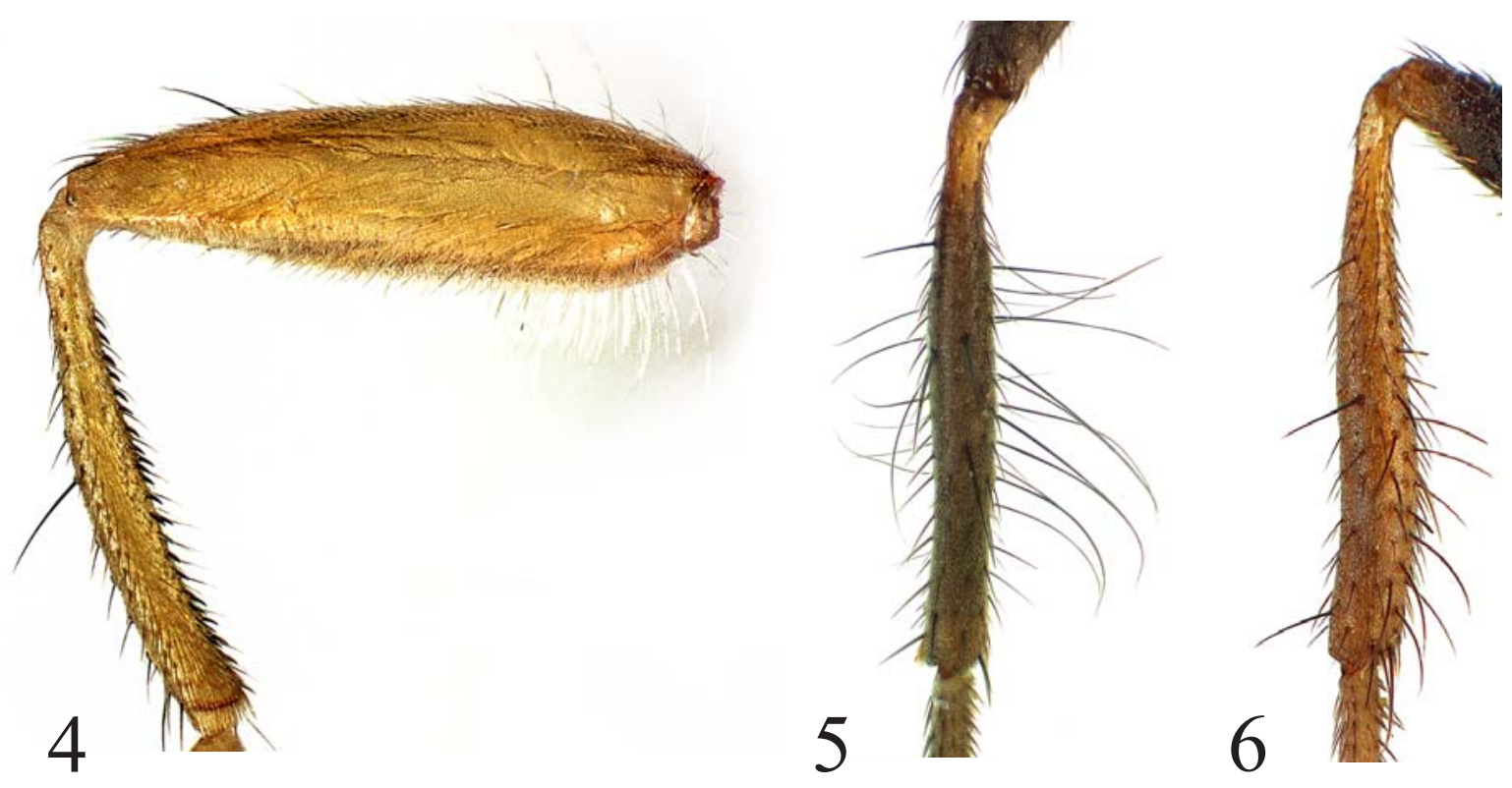

Figs 4-6. Male legs of Microprosopa: 4 - M. pallidicauda (Zetterstedt), fore leg, anterior view; $5-$ M. crinipes Ringdahl, hing tibia, anterodorsal view; $6-M$. taimyrica sp.n., HT, hind tibia, anterior view.

Рис. 4-6. Ноги самцов Microprosopa: $4-$ M. pallidicauda (Zetterstedt), передняя нога, спереди; 5 - M. crinipes Ringdahl, голень задней ноги, антеродорсально; 6 - M. taimyrica sp.n., голотип, голень задней ноги, спереди.

with $0-1$ apical anterodorsal and 1 apical anteroventral setae; in M. crinipes Ringdahl, 1928 and M. taimyrica sp.n. additionally with long setae on anteroventral surface in male (Figs 5, 6).

Wing clear or slightly darkened (Fig. 7-9), in $M$. taimyrica sp.n. darkened along longitudinal veins (Fig. 10 ); vein $\mathrm{R}_{1}$ bare on apical third of dorsal surface. Calypters, margins of calypters, and halteres yellowish.

Abdomen cylindrical, black, greyish dusted, covered with rare yellow and black hairs. Tergites 3-6 each usually with a row of marginal setae. Male sternite 4 simple (Figs 11-22). Male sternite 5 with two lobes, each may be simple (Figs 28-33) or branchy (Figs 23-27, 34-38), symmetrical (Figs 23, 24, 28-33, 37,38 ) or asymmetrical (Figs 25-27, 4-36). Male cerci small, simple; surstyli elongate (Figs 39-62).

Ovipositor short, cylindrical, with the proctiger shifted dorsally (Figs 65). Female tergite 7 divided medially into two sclerites, each triangular in lateral view; tergite 8 also divided medially into two sclerites, excluding $M$. unguiculata (Malloch, 1919). Sternite 7 simple (Fig. 64, $66,68,69,71-77)$ or its lateral margins fused with lateral margins of sternite 7 forming syntergosternite as in M. crinipes (Fig. 63, 67) and M. heteromyzina (Zetterstedt, 1838) (Fig. 70). Sternite 8 divided medially into two sclerites. 1838)

Microprosopa (Allomyella) albipennis (Zetterstedt,

Figs $11,23,39,40,66,78$.

albipennis Zetterstedt, 1838: 729 (Cordylura). Type-locality: "Lapponia Umensi... in insula illa parva Dolpatie in lacu StorUman... (Lappon)" (Sweden). niveipalpis Zetterstedt, 1846: 2044 (Cordylura). Type-localities: "alpibus Darlecarlicis ... Jemtlandia ... Lapponia Lulensi ... Norwegia ... Suul Waerdaliae" (Sweden, Norway).

The species was mentioned by Gorodkov for the European part of Russia [Gorodkov, 1986: 24] and Siberea [Gorodkov, 1970: 455] without indicating a specific locality, also by Šifner [2008: 137] for Moscow Oblast.

MATERIAL EXAMINED. Karelia: Puykolla $\left(62.0509^{\circ} \mathrm{N}\right.$ 30.6632 ${ }^{\circ}$ E), 15.V.-16.VI.1991, Polevoy (1 $\sigma^{7}$, ZMUM); Tyumen' Oblast: Sob' env. (67.07N 65.46E), 26-31.VII.2011, K. Tomkovich (1 ア', ZMUM);

ADDITIONAL MATERIAL EXAMINED. Holotype $\sigma^{7}$ of $\mathrm{Mi}$ croprosopa albipennis (Zetterstedt), with labells: 1) small black tag, 2) "C. albipennis O' Dolpatie", 3)"Holotypus Cordylura albipennis Zett. Labelled 1954 by J.R. Vockeroth” (MZLU). Finland: Kilpisjärvi, 10.VII.1935, Hellén (1 q, MZH).

DESCRIPTION. Male, female. Small-sized flies (3.1-3.2 mm long).

Head. Frontal vitta black, only in lower third or half yellow, matt; fronto-orbital plate and ocellar triangle black, greyish dusted. Face from whitish to blackish. Parafacial yellowish. Gena yellowish or brownish; postgena black, greyish dusted. Postcranium black, greyish dusted, covered with black setae in upper third and pale hairs in lower part. 3 orbitals, 2-3 frontals, 1 ocellar, 1 small postocellar, 1 inner vertical, 1 outer vertical; 1-2 pairs of pale vibrissae present. Antenna black, but pedicel sometimes partly brownish. Postpedicel rounded apically, approximately 2 times as long as wide. Arista black, basally thickened, bare. Clypeus and proboscis black. Palpus broadened towards apex, yellow.

Thorax and scutellum black, greyish dusted. Acrostical hairs in two rows, dorsocentrals $3+3$, intra-alars $1+2$, supra-alars $1+2$, postpronotals 2 , notopleurals 2 , 

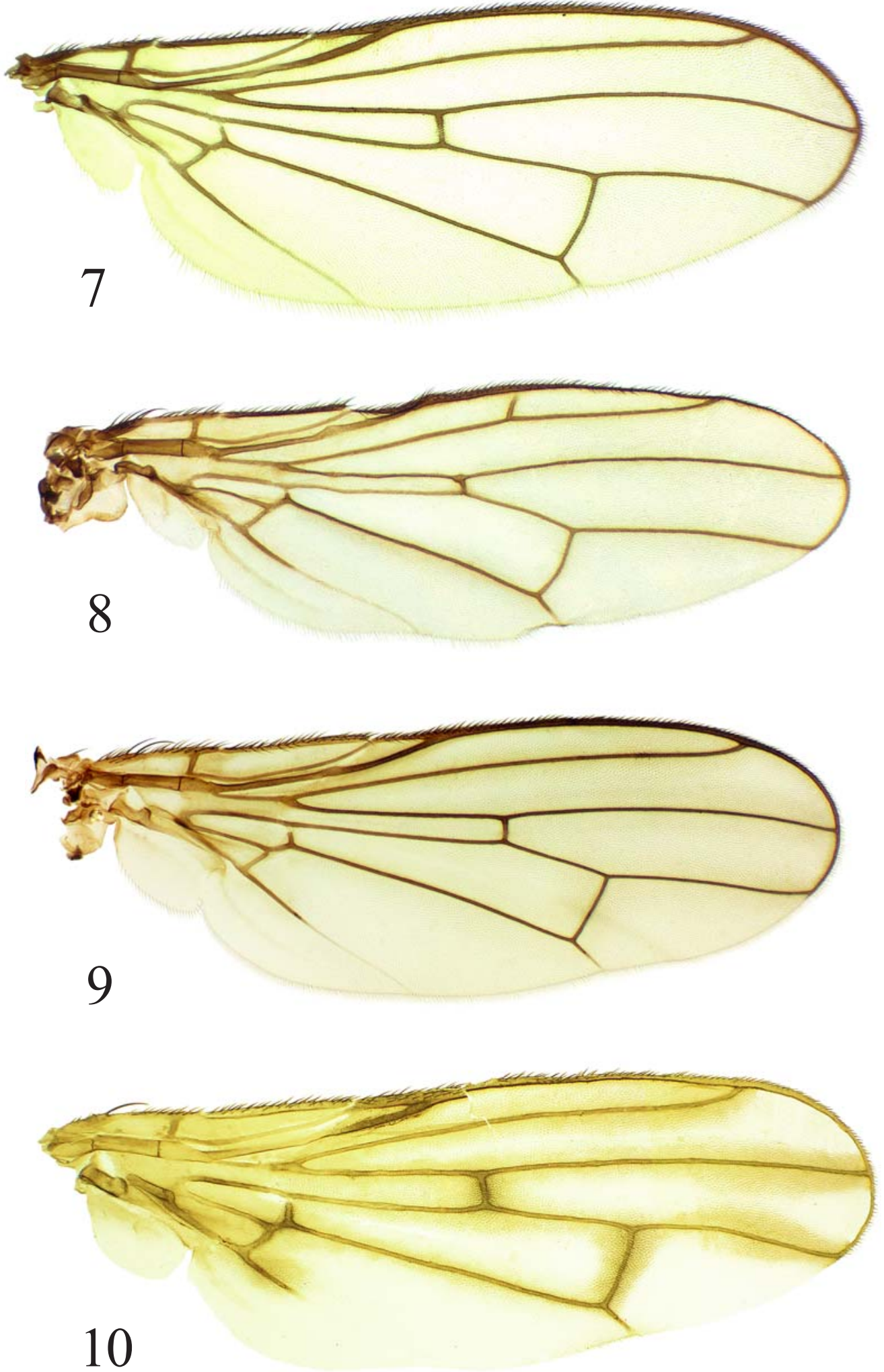

Figs 7-10. Wing of Microprosopa: 7 - M. crinipes Ringdahl; $8-$ M. frigida (Holmgren); $9-$ M. pallidicauda (Zetterstedt); $10-$ M. taimyrica sp.n.

Рис. 7-10. Крыло Microprosopa: 7 - M. crinipes Ringdahl; 8 - M. frigida (Holmgren); 9 - M. pallidicauda (Zetterstedt); $10-$ M. taimyrica sp.n. 
postalars 2. Proepisternum with hairs in central part, with 1-2 pale setulae near lower margin. Proepimeron with one pale setula. Anepisternum covered with pale hairs in posterior half, with 1-2 black setae along posterior margin. Katepisternum covered with pale hairs in posterior half and 1 strong seta in upper posterior corner. Anepimeron usually with one or several hairs. Scutellum black, greyish dusted, with a pair of basal scutellar and a pair of apical scutellar setae.

Coxae, trochanters and femora of all legs black; tibiae and tarsi of all legs yellow. Fore femur with a row of posterodorsal setae, with pale hairs posteroventrally. Fore tibia with short black spinules ventrally in two rows on whole length, with 1 dorsal seta near middle, and with 1 preapical dorsal seta. Mid femur with irregular row of pale setae anteriorly, with 1 preapical posterior and $0-1$ preapical posterodorsal setae, also with pale hairs ventrally. Mid tibia with 1 anterodorsal and $0-1$ posterodorsal setae near middle, also with a ring of apicals. Hind femur with a row of black or pale anterodorsal and a row of pale anteroventral setae. Hind tibia with 1-2 anterodorsal, 1 posterodorsal and 1 preapical dorsal setae, also with 1 apical anteroventral seta.

Wing clear or slightly milky; veins brownish. $\mathrm{R}_{1}$ bare dorsally. Calypters, margins of calypters, and halteres whitish or yellowish.

Abdomen black, subshining, tergites 3-5 shining along lateral margins, female tergite 6 shining completely. Male sternite 4 rectangular, about 2 times as wide as long (Fig. 11). Male sternite 5 symmetrical, lobes bifurcate (Fig. 23). Epandrium and surstyli as in Figs 39, 40. Female sternites 5-7 as in Fig. 66.

DISTRIBUTION. Russia (Fig. 78): Karelia, Moscow Oblast, Tyumen' Oblast. — Europe (Scandinavia).

Microprosopa (Allomyella) crinipes Ringdahl, 1928 Figs 5, 7, 12, 24, 41, 42, 63, 67, 79.

crinipes Ringdahl, 1928: 21 (Microprosopa). Type-locality: "Ufer von Tjuonajaure im nördlichen Lappland in Regio arctica, 1000 M."

The species was recorded for Russia from Chukotka [Ozerov \& Krivosheina, 2014: 206].

MATERIAL EXAMINED. Chukotka: Meynypil'gyno env. $\left(62.555^{\circ} \mathrm{N} 177.0633^{\circ} \mathrm{E}\right), 29 . \mathrm{VII} .2015$, P.S. Tomkovich (1 o', 1 t ZMUM); Krasnoyarsk Kray: Agapa (ca. $71.412^{\circ} \mathrm{N} 89.2689^{\circ} \mathrm{E}$ ), the Pyasina River, 15.VII.1967, Gorodkov (1 $\sigma^{7}$, ZISP); Lake Engel'gardt $\left(75.101^{\circ} \mathrm{N} 100.2344^{\circ} \mathrm{E}\right), 2$.VIII.1967, Gorodkov (1 + , ZISP); Dikson I. (73.5042 $\left.{ }^{\circ} \mathrm{N} 80.3251^{\circ} \mathrm{E}\right), 30$. VII.1948, Korotkevich $\left(1\right.$ ㅇ, ZISP); Ust'-Tareya $\left(73.2527^{\circ} \mathrm{N} 90.5962^{\circ} \mathrm{E}\right), 24$.VII. and 4.VIII.1967, K. Gorodkov (11 $\sigma^{\top} \sigma^{\top}, 6$ 9 , ZISP); Tyumen' Oblast: $85 \mathrm{~km}$ WSW of Antipayuta (ca. $68.8208^{\circ} \mathrm{N} 75.3442^{\circ} \mathrm{E}$ ), 31.VII 1977, Gorodkov ( $1 \sigma^{7}, 5$ 90 , ZISP); the upper reaches of the Pura River (ca. $\left.66.3202^{\circ} \mathrm{N} 77.989^{\circ} \mathrm{E}\right), 10 \mathrm{~km} \mathrm{NW}$ of Lake Barasmu, 21 VII.1967, Gorodkov (3 $\sigma^{7} \sigma^{7}, 1$ ․, ZISP); Yakutia: Sukharnoe [=Ambarchik] env. $\left(69.6258^{\circ} \mathrm{N} 162.3029^{\circ} \mathrm{E}\right), 13$.VII.1905, Buturlin ( $\sigma^{7}$, ZISP); Stolb I. (72.3956 $\left.{ }^{\circ} \mathrm{N} 126.6588^{\circ} \mathrm{E}\right)$, Lena delta, 25 VII.1957, Gorodkov (1 $\sigma^{7}$, ZISP); Pokhodsk $\left(69.079^{\circ} \mathrm{N} 160\right.$ $\left.9615^{\circ} \mathrm{E}\right), 18$.VII.1973, Vinokurov (1 $\bigcirc^{\top}, 4$ 우,ZISP); Uryung-Khaya $\left(72.8124^{\circ} \mathrm{N} 113.2316^{\circ} \mathrm{E}\right), 4$ and 8.VIII.1988, Gorodkov (2 $\sigma^{\top} \sigma^{7}, 3$ oㅇ, ZISP); Chay-Tumus $\left(72.3166^{\circ} \mathrm{N} 125.7333^{\circ} \mathrm{E}\right), 27 . \mathrm{VII} .1957$, Gorodkov (1 $\sigma^{7}$, ZISP); Chokurdakh $\left(70.6187^{\circ} \mathrm{N} 147.8958^{\circ} \mathrm{E}\right)$,

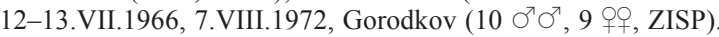

DESCRIPTION. Male, female. Small or mediumsized flies (3.8-5.6 mm long).

Head. Frontal vitta black, only in lower third or quarter yellow, greyish dusted; fronto-orbital plate and ocellar triangle black, greyish dusted. Face yellowish completely or darkened in upper half. Parafacial yellow. Gena yellow, postgena black. Postcranium black, greyish dusted, covered with black setae in upper third and pale hairs in lower part. 3 orbitals, 2-3 frontals, 1 ocellar, 1 small postocellar, 1 inner vertical, 1 outer vertical; 1 or 2 pairs of vibrissae present. Antenna black. Postpedicel rounded apically, approximately 2 times as long as wide. Arista black, basally thickened, bare. Clypeus and proboscis black. Palpus broadened towards apex, yellow.

Thorax and scutellum black, greyish dusted. Acrostical hairs in two irregular rows, dorsocentrals (3-4)+3, intra-alars $1+2$, supra-alars $1+2$, postpronotals 2 , notopleurals 2, postalars 2. Proepisternum with hairs in central part, with 1-2 long pale setae near lower margin. Proepimeron with 1-2 long pale setae. Anepisternum covered with pale hairs in posterior half, with 2-4 black setae along posterior margin. Katepisternum covered with pale hairs in posterior half and 1 strong seta in upper posterior corner. Anepimeron usually with a few hairs. Scutellum black, greyish dusted, with a pair of basal scutellar and a pair of apical scutellar setae.

Legs black, but fore and mid tibiae, all tarsi, and sometimes hind tibia yellowish. Fore femur with rows of posterodorsal and posteroventral setae, with pale hairs posteriorly and posteroventrally. Fore tibia with short black spinules ventrally in two rows on whole length, with 1 dorsal and 1 posterior setae near middle, and with 1 preapical dorsal seta. Mid femur with irregular row of anterior setae in apical half and a row of posteroventral setae, with 1 preapical posterior and 1 preapical posterodorsal setae. Mid tibia with 1 anterodorsal, 1 posterodorsal and 1 posterior setae near middle, and a ring of apicals. Hind femur with a row of black or pale anterodorsal and rows of pale anteroventral and posteroventral setae. Hind tibia with 2 anterodorsal, 1-2 posterodorsal, 1 preapical dorsal, and 1 apical anteroventral setae; additionaly with long setae on anterior, posterior and ventral surfaces in male (Fig. 5) and several short anteroventral setae at middle in female.

Wing clear or slightly darkened; veins blackish. $\mathrm{R}_{1}$ bare dorsally (Fig. 7). Calypters, margins of calypters, and halteres yellowish.

Abdomen black greyish dusted, tergites 3-5 shining along lateral margins. Male sternite 4 rectangular, about 2.5 times as wide as long (Fig. 12). Male sternite 5 symmetrical, lobes bifurcate apically (Fig. 24). Epandrium and surstyli as in Figs 41, 42. Female sternites 5-7 as in Figs 63, 67; lateral margins of sternite 7 fused with lateral margins of sternite 7 forming syntergosternite; sternite 7 about 2 times as long as tergite 5 .

DISTRIBUTION. Russia (Fig. 79): Chukotka, Krasnoyarsk Kray, Tyumen' Oblast, Yakutia. — Europe ([?]Andorra, Sweden), North America. 

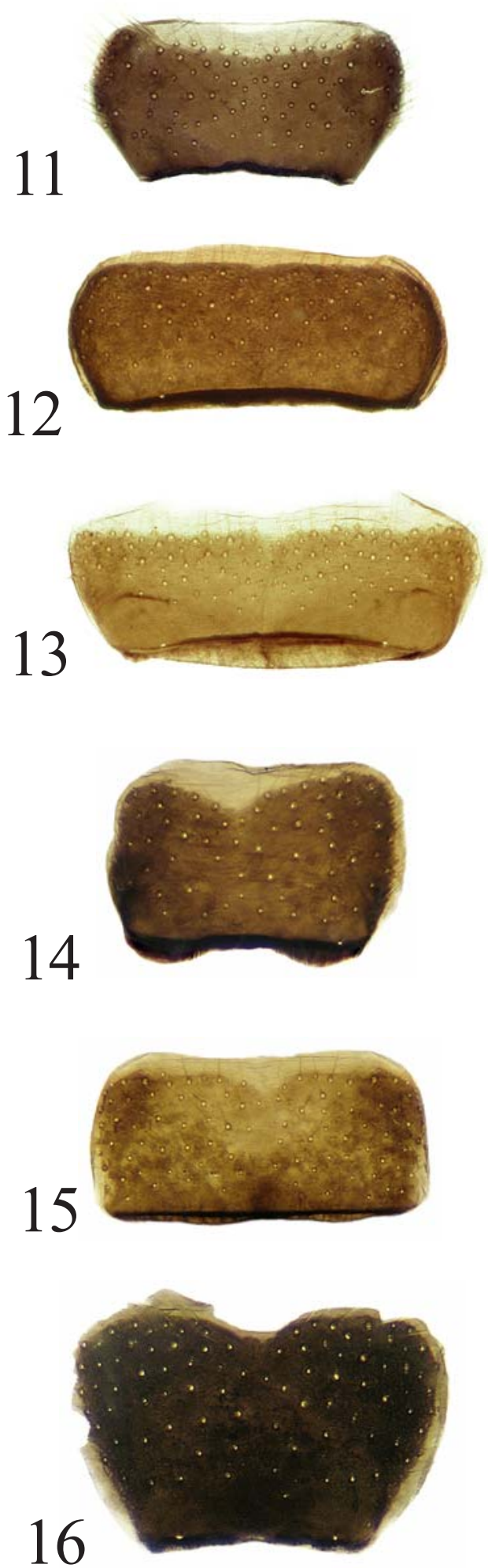
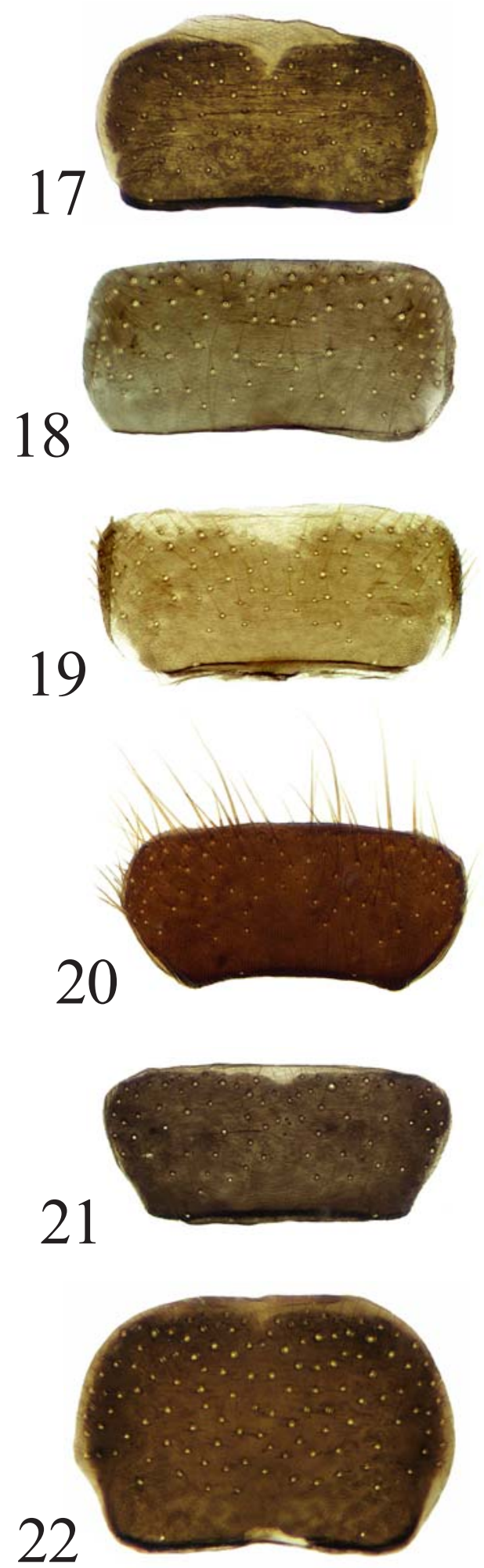

Figs 11-22. Male sternite 4 of Microprosopa: $11-$ M. albipennis (Zetterstedt); $12-$ M. crinipes Ringdahl; $13-$ M. frigida (Holmgren); $14-$ M. haemorrhoidalis (Meigen); $15-$ M. heteromyzina (Zetterstedt); $16-$ M. lineata (Zetterstedt); $17-M$. pallidicauda (Zetterstedt); $18-$ M. paveli Ozerov et Krivosheina; $19-$ M. portenkoi Stackelberg; $20-$ M. taimyrica sp.n.; $21-M$. unguiculata (Malloch); $22-$ M. zlobini Ozerov.

Рис. 11-22. Стернит 4 самца Microprosopa: $11-$ M. albipennis (Zetterstedt); $12-$ M. crinipes Ringdahl; $13-$ M. frigida (Holmgren); $14-$ M. haemorrhoidalis (Meigen); $15-$ M. heteromyzina (Zetterstedt); $16-$ M. lineata (Zetterstedt); $17-M$. pallidicauda (Zetterstedt); $18-$ M. paveli Ozerov et Krivosheina; $19-$ M. portenkoi Stackelberg; $20-$ M. taimyrica sp.n.; $21-M$. unguiculata (Malloch); $22-$ M. zlobini Ozerov. 
Microprosopa (Allomyella) frigida (Holmgren, 1883)

Figs 2, 8, 13, 25-27, 43, 44, 68, 80.

frigida Holmgren, 1883: 176 (Cordylura). Type-locality: Matochkin Schar (ca. 73.375N 55.0964E), Novaya Zemlya (Russia). varitibia Becker, 1897: 400 (Microprosopa). Type-locality: Malye Karmakuly $\left(72.3714^{\circ} \mathrm{N} 52.7415^{\circ} \mathrm{E}\right)$, Novaya Zemlya (Russia).

Besides type-locality (Novaya Zemlya) noted for Russia from Kola peninsula [Hackman, 1956: 58, as M. portenkoi (Stackelberg)], Krasnoyarsk Kray [Ozerov \& Barkalov, 2014: 561 (as Allomyella)] and Yakutia [Engelmark, 1999: 159].

MATERIAL EXAMINED. Arkhangelsk Oblast: Novaya Zemlya, Matochkin Shar (ca. $\left.73.375^{\circ} \mathrm{N} 55.0964^{\circ} \mathrm{E}\right), 1-5$. VIII.1889, K. Nosilov (1 9 , ZISP); same place, 29.VI--1.VIII.1925, Vakulenko (4 $\sigma^{7} \sigma^{7}, 9$ 우, ZISP); Pushlakhta $\left(64.8107^{\circ} \mathrm{N} 36.5779^{\circ} \mathrm{E}\right)$, 17.VII.1904, Sokolovskiy (1 O', ZISP); Krasnoyarsk Kray: 104 km NNW of Noril'sk, the Nizhnyaya Agapa River (ca. $70.0972^{\circ} \mathrm{N}$ $\left.86.6883^{\circ} \mathrm{E}\right), 24-25$. VII.1973, Sukacheva, Zherikhin (2 $0^{7} 0^{7}$, ZISP); Agapa (ca. $71.412^{\circ} \mathrm{N} 89.2689^{\circ} \mathrm{E}$ ), the Pyasina River, $14-15$.VII 1967, Gorodkov ( $8 \mathrm{O}^{7} \mathrm{O}^{7}, 3$ 우, ZISP); Taymyr, Lake Taymyr (74. $0775^{\circ} \mathrm{N} 101.2808^{\circ} \mathrm{E}$ ), west bank W of Baykura-Neru, 28.VII.1976, A. Rasnitsyn, I. Sukacheva (2 $O^{7} O^{7}$, ZMUM); Taymyr, Taymyrskiy Reserve, Cordon Ary-Mas ( $\left.72.5^{\circ} \mathrm{N} 101.94^{\circ} \mathrm{E}\right), 14 \mathrm{~m}, 9-10$. VII.2010, A. Barkalov (9 $\mathrm{O}^{7} \mathrm{O}^{\mathrm{T}}$, ISEA and ZMUM); Ust'-Tareya $\left(73.2527^{\circ} \mathrm{N}\right.$ 90.5962 ${ }^{\circ}$ ), 24.VII.1967, Gorodkov (1 $\sigma^{7}$, ZISP); Tyumen' Oblast: Neroyka env. (ca. 64.57N 59.67E), $950 \mathrm{~m}$, 6.VII.1990, Malozemov (2 $\mathrm{O}^{7} \mathrm{O}^{\top}$, ZISP); Yakutia: Tiksi env. (71.6351 $\left.{ }^{\circ} \mathrm{N} 128.8579^{\circ} \mathrm{E}\right)$, 9.VII.1957, Gorodkov (1 O $\sigma^{7}$, ZISP).

DESCRIPTION. Male (Fig. 2), female. Small or medium-sized flies (3.6-4.3 mm long).

Head. Frontal vitta black, only in lower third or quarter yellow, matt; fronto-orbital plate and ocellar triangle black, greyish dusted. Face blackish in upper half and yellowish in lower half. Parafacial blackish. Gena black completely or yellow in lower half, postgena black, greyish dusted. Postcranium black, greyish dusted, covered with black setae in upper third and with pale hairs in lower part. 3 orbitals, 2-3 frontals, 1 ocellar, 1 small postocellar, 1 inner vertical, 1 outer vertical; 1-2 pairs of pale vibrissae present. Antenna black. Postpedicel more or less rounded apically, approximately 2 times as long as wide. Arista black, basally thickened, bare. Clypeus and proboscis black. Palpus broadened towards apex, yellow.

Thorax and scutellum black, greyish dusted. Acrostical hairs in two rows, dorsocentrals (3-4)+3, intraalars $1+2$, supra-alars $1+2$, postpronotals 2 , notopleurals 2, postalars 2. Proepisternum with hairs in central part, with 1-2 pale setulae near lower margin. Proepimeron with one pale setula. Anepisternum covered with pale hairs in posterior half, with 1-2 black setae along posterior margin. Katepisternum covered with pale hairs in posterior half and 1 strong seta in upper posterior corner. Anepimeron usually with one or several hairs, but sometimes bare. Scutellum black, greyish dusted, with a pair of basal scutellar and a pair of apical scutellar setae.

Coxae, trochanters and femora of all legs black; tibiae and tarsi of all legs yellow, sometimes hind femur darkened. Fore femur with a row of posterodorsal setae in apical half, with pale hairs posteroventrally.
Fore tibia with short black spinules ventrally in two rows on whole length, with 1 dorsal and 1 posterior setae near middle, with 1 preapical dorsal seta and 1 apical posterior seta. Mid femur with irregular row of anterior setae in apical half, with 1 preapical posterior and 1 preapical posterodorsal setae, also with pale hairs ventrally. Mid tibia with 1 anterodorsal, 1 posterodorsal, and $0-1$ posterior setae near middle, also with a ring of apicals. Hind femur with a row of black anterodorsal and a row of pale anteroventral setae. Hind tibia with 2 anterodorsal, 1-2 posterodorsal, 1 preapical dorsal, and 1 preapical posteroventral setae, also with 1 apical anteroventral seta.

Wing (Fig. 8) slightly darkened; veins blackish. $\mathrm{R}_{1}$ bare dorsally. Calypters, margins of calypters, and halteres whitish or yellowish.

Abdomen black, greyish dusted, sometimes male tergites 4-5 shining along lateral margins. Male sternite 4 rectangular, about 2.5 times as wide as long (Fig. 13). Male sternite 5 asymmetrical, left lobe with three horns, right lobe bifurcate (Figs 25-27). Epandrium and surstyli as in Figs 43, 44. Female sternites 5-7 as in Fig. 68; sternites 6 and 7 poorly sclerotized in median part.

DISTRIBUTION. Russia (Fig. 80): Arkhangelsk Oblast, Krasnoyarsk Kray, Tyumen' Oblast, Yakutia. - Europe (Sweden), North America.

Microprosopa (Microprosopa) haemorrhoidalis (Meigen, 1826)

Figs $14,28,45,46,69,81$.

haemorrhoidalis Meigen, 1826: 237 (Cordylura). Type-locality: "Schweden".

pallipes Zetterstedt, 1838: 732 (Cordylura). Type-localities: "Lapponia passim; inter Tresiinda et Naestansjo ut et ad Lycksele, Lapponiae Umensis ... Jiickasjervi, Lapponiae Tornensis (Lapponia; Scania)".

lividipes Zetterstedt, 1846: 2042 (Cordylura). Typelocality: "Lapponia Lulensi ad Qvickjock" (Sweden).

Reported for Russia from Kola Peninsula [Hackman, 1956: 57], Siberia (Krasnoyarsk Kray, Sayan Mountains) and Far East [Gorodkov, 1986: 25; Ozerov \& Barkalov, 2014: 562; Ozerov \& Krivosheina, 2014: 213].

MATERIAL EXAMINED. Arkhangelsk Oblast: Khal'mer'yu (ca. $\left.67.5507^{\circ} \mathrm{N} 53.962^{\circ} \mathrm{E}\right)$, 19.VII.1962, Gorodkov $\left(2 \mathrm{O}^{7} \sigma^{7}, 1\right.$ \% ZISP); Nar'yan-Mar $\left(67.6317^{\circ} \mathrm{N} 52.9857^{\circ} \mathrm{E}\right)$, 12.VII.2008, A.L. Ozerov (1 $0^{\top}$, ZMUM); Nar'yan-Mar $\left(67.6369^{\circ} \mathrm{N} 53.0316^{\circ} \mathrm{E}\right), 2$ and 4.VIII.1978, Gorodkov (2 $0^{7} \sigma^{7}$, ZISP); Buryatia: Barguzinskiy Reserve (ca. $54.35^{\circ} \mathrm{N} 109.51^{\circ} \mathrm{E}$ ), 23.VII.1965, Negrobov (1 + , ZISP); Chukotka: $20 \mathrm{~km}$ NNE of Iul'tin $\left(67.7244^{\circ} \mathrm{N} 178.5158^{\circ} \mathrm{W}\right)$, 22.VII. 1963 , Gorodkov (1 $0^{7}, 1$ \%, ZISP); $5 \mathrm{~km} \mathrm{~N}$ of Egvekinot $\left(66.3637^{\circ} \mathrm{N}\right.$ 179.1165 ${ }^{\circ}$ ), 26.VII.1963, Gorodkov (1 $\sigma^{7}$, ZISP); bank of the Anadyr River $\left(64.72^{\circ} \mathrm{N} 175.21^{\circ} \mathrm{E}\right), 25$. VI.-19.VII.2014, A. Barkalov (1 $0^{\gamma}$, in ISEA); Apapel'khin (ca. $\left.69.7644^{\circ} \mathrm{N} 170.6176^{\circ} \mathrm{E}\right)$, 1.VII. 1972, Gorodkov (1 9 , ZISP); same place, 15.VII.1963, Gorodkov (2 $\sigma^{7} \sigma^{\prime \prime}, 3$ 우, ZISP); Iul'tin $\left(67.8669^{\circ} \mathrm{N} 178.7333^{\circ} \mathrm{W}\right), 20 . \mathrm{VII} .1963$, Gorodkov ( $10^{7}$, ZISP); Komsomol'skiy $\left(69.1309^{\circ} \mathrm{N} 172.7358^{\circ} \mathrm{E}\right)$,

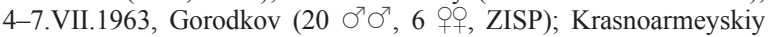
$\left(69.5435^{\circ} \mathrm{N} 171.9944^{\circ} \mathrm{E}\right), 3$ and 8.VII.1963, Gorodkov (12 $0^{7} \sigma^{7}, 4$ OO, ZISP); Pevek $\left(69.7022^{\circ} \mathrm{N} 170.2982^{\circ} \mathrm{E}\right), 1,11$, and 12.VII.1963, 4.VIII.1972, Gorodkov (24 $\mathrm{O}^{7} \mathrm{O}^{7}, 10$ 우, ZISP); Polyarnyy mine (69.

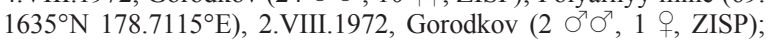
Chaunskaya Bay, the mouth of the Ichun' River $\left(68.8541^{\circ} \mathrm{N} 170\right.$. $\left.5476^{\circ} \mathrm{E}\right), 8$.VI. and 3.VII.1963, Semenov (4 ㅇ, ZISP); Irkutsk 


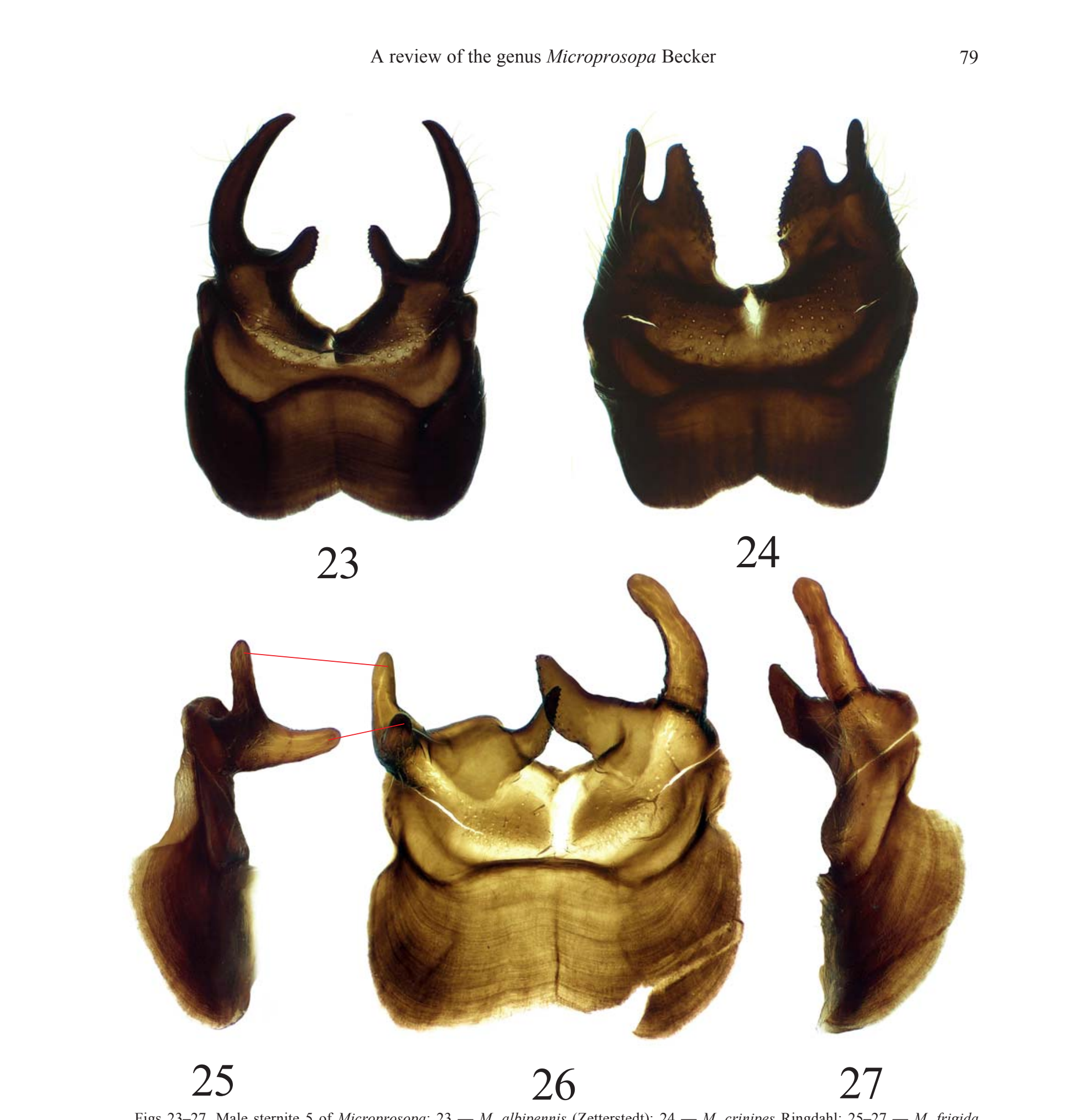




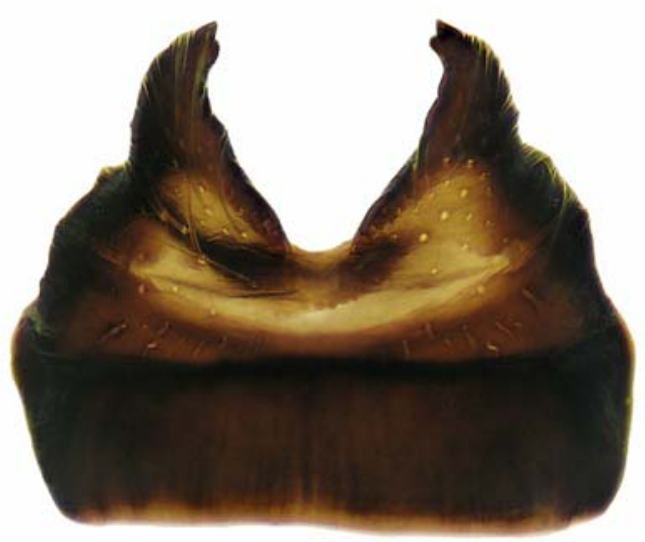

28
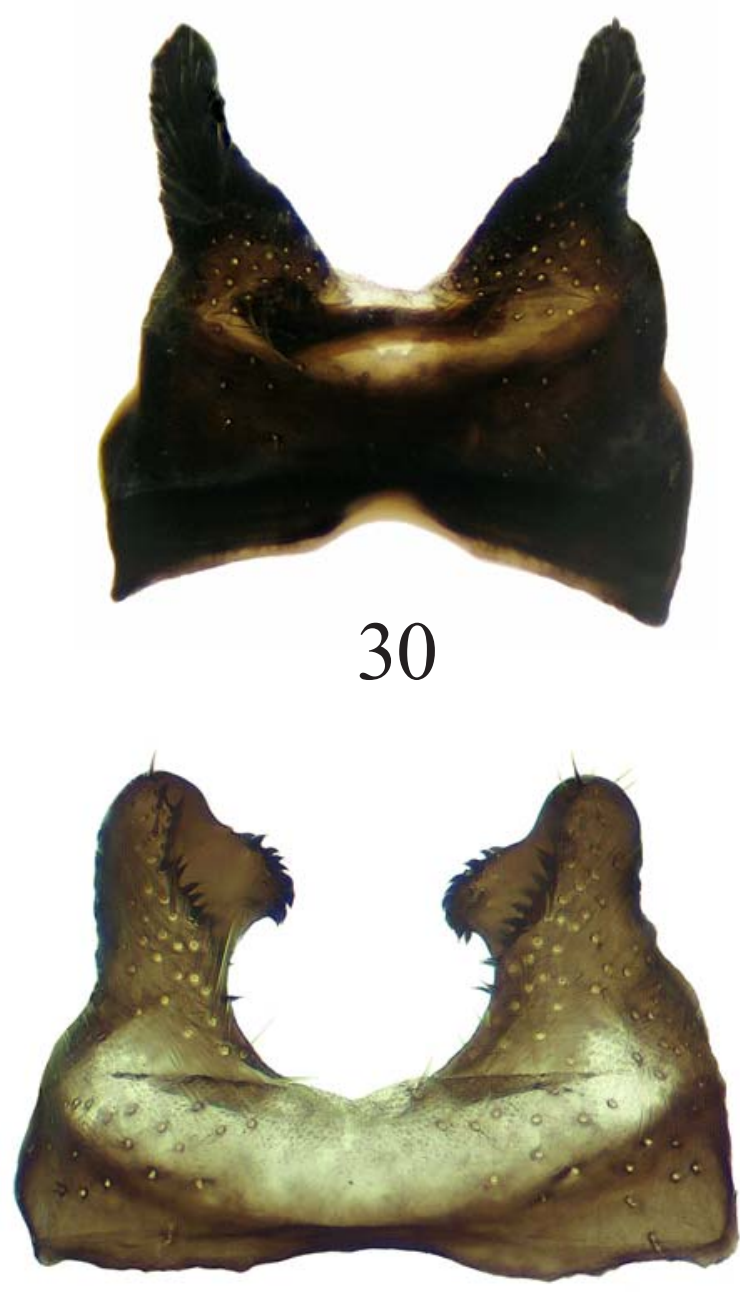

32

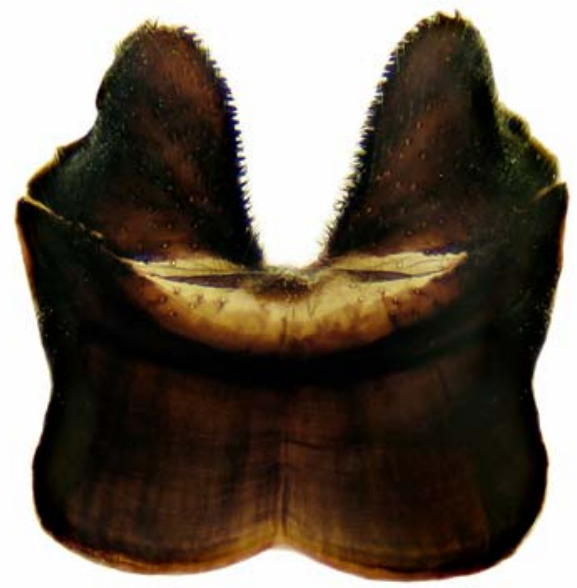

29

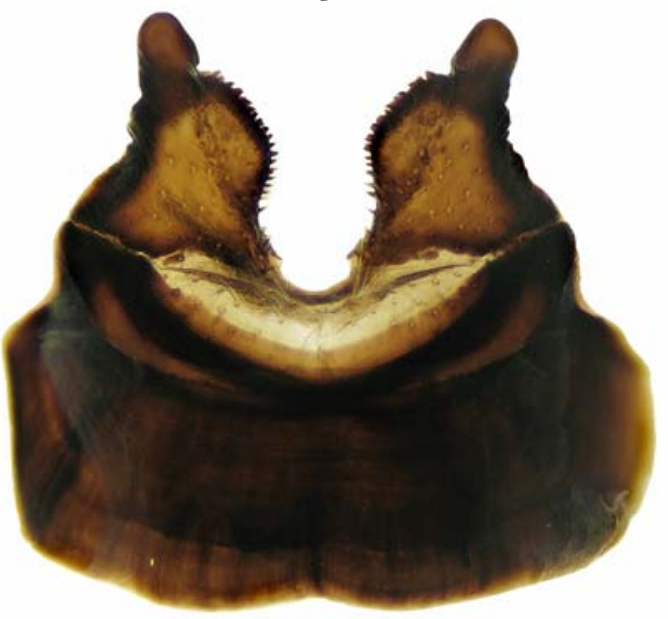

31

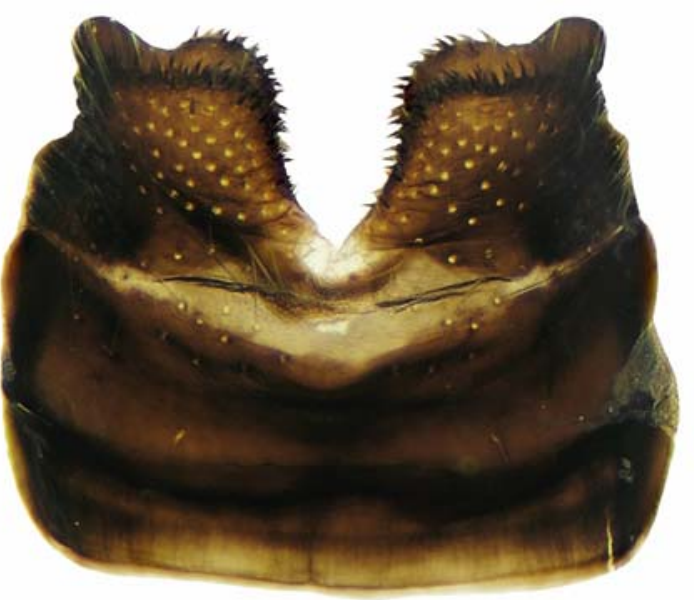

33

Figs 28-33. Male sternite 5 of Microprosopa: $28-M$. haemorrhoidalis (Meigen); $29-$ M. heteromyzina (Zetterstedt); $30-M$. lineata (Zetterstedt); $31-$ M. pallidicauda (Zetterstedt); $32-$ M. paveli Ozerov et Krivosheina; $33-$ M. zlobini Ozerov.

Рис. 28-33. Стернит 5 самца Microprosopa: $28-$ M. haemorrhoidalis (Meigen); $29-$ M. heteromyzina (Zetterstedt); $30-M$. lineata (Zetterstedt); $31-$ M. pallidicauda (Zetterstedt); $32-$ M. paveli Ozerov et Krivosheina; $33-$ M. zlobini Ozerov. 


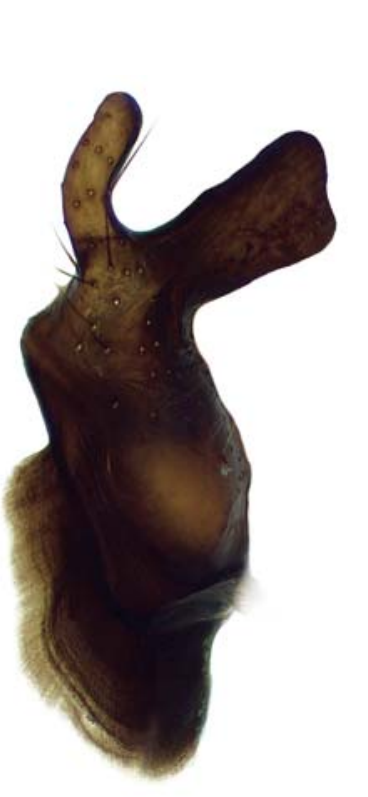

34

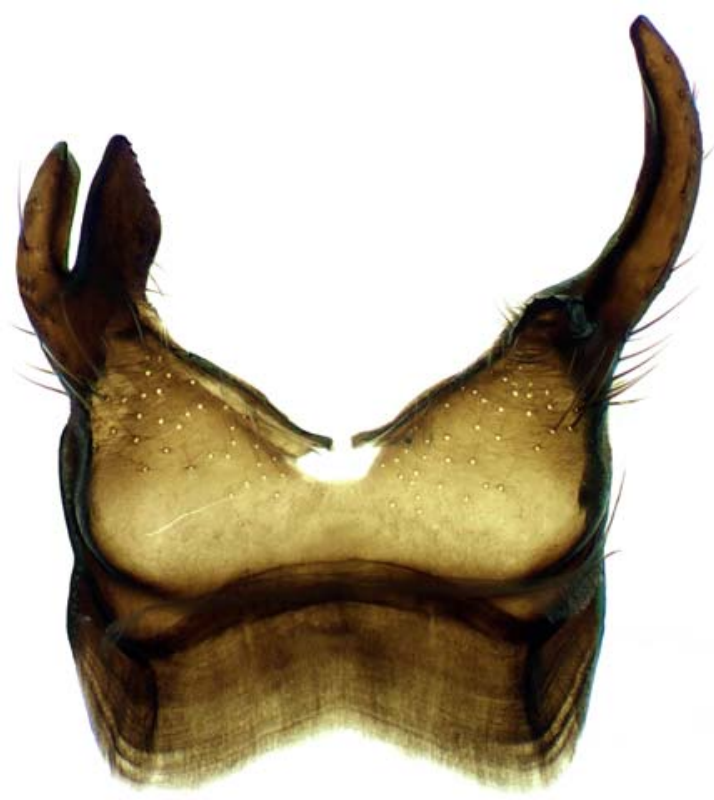

35
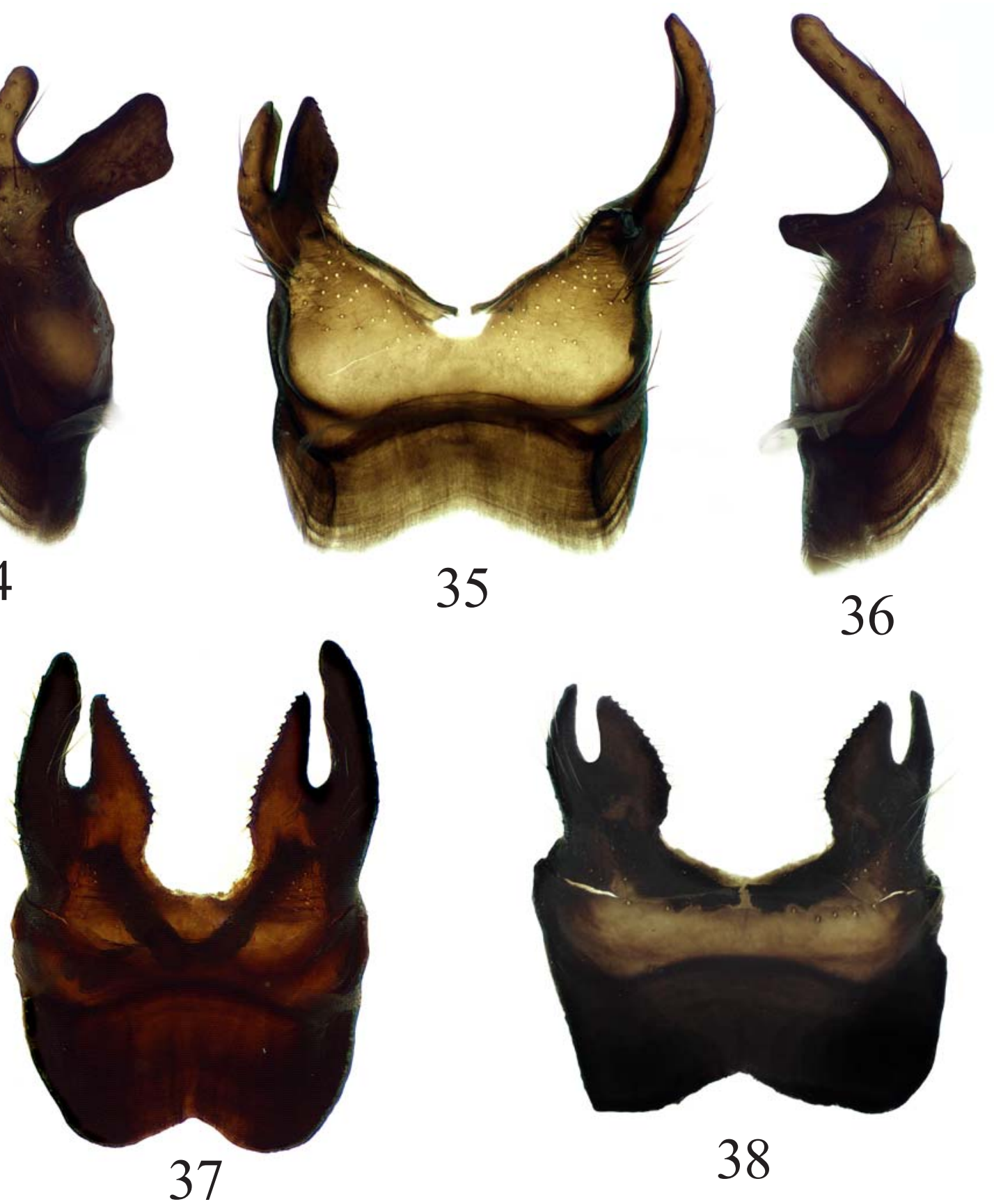

Figs 34-38. Male sternite 5 of Microprosopa: 34-36 - M. portenkoi Stackelberg; $37-$ M. taimyrica sp.n.; $38-$ M. unguiculata (Malloch).

Рис. 34-38. Стернит 5 самца Microprosopa: 34-36 - M. portenkoi Stackelberg; $37-$ M. taimyrica sp.n.; $38-$ M. unguiculata (Malloch).

the Enisey River, 1.VII.1967, Gorodkov (1 $0^{7}$, ZISP); Medvezhiy $\left(69.2845^{\circ} \mathrm{N} 88.1488^{\circ} \mathrm{E}\right)$, Noril'sk env., 6.VII.1967, Gorodkov (1 ZISP); Noril'sk $\left(69.3396^{\circ} \mathrm{N} 88.2147^{\circ} \mathrm{E}\right), 6$ and 9 .VII.1967, Gorodkov (13 $\mathrm{O}^{7} \mathrm{O}^{3}, 10 \mathrm{O}+\mathrm{O}$, ZISP); Glubokoe Lake, $60 \mathrm{~km}$ O of Noril'sk (ca. $\left.69.373^{\circ} \mathrm{N} 89.799^{\circ} \mathrm{E}\right)$, 8.VII.1967, Gorodkov (1 ○ $^{7}$, ZISP); Noril'sk env., Talnakh $\left(69.5003^{\circ} \mathrm{N} 88.4476^{\circ} \mathrm{E}\right), 22$. VIII.1973, Gorodkov (1 , ZISP); Turukhansk $\left(65.7971^{\circ} \mathrm{N} 87.9527^{\circ} \mathrm{E}\right)$, 28.VI. 1967, Gorodkov (19 $\mathrm{O}^{7} \mathrm{O}^{7}$, ZISP); Ust'-Tareya $\left(73.2527^{\circ} \mathrm{N} 90\right.$ $\left.5962^{\circ} \mathrm{E}\right), 29$.VII. 1967 , Gorodkov ( $1 \mathrm{O}^{7}$, ZISP); Khatanga $\left(71.9815^{\circ} \mathrm{N}\right.$ $\left.102.4832^{\circ} \mathrm{E}\right), 25 . \mathrm{VII} .1971$, Chelnokov $\left(2 \mathrm{O}^{\top} \mathrm{O}^{\top}\right.$, ZISP); same place, 25.VIII.1971, Gorodkov (17 $0^{7} \bigcirc^{7}, 2$ 우, ZISP); Leningrad Oblast:
Gatshina $\left(59.56^{\circ} \mathrm{N} 30.13^{\circ} \mathrm{E}\right), 15 . \mathrm{V} .1903$, Barovskiy (1 을 ZISP); Jukki (ca. $60.11^{\circ} \mathrm{N} 30.29^{\circ}$ E), 11.VI.1931, 12.VI.1932, A. Stackelberg ( $2 \bigcirc^{\top} \sigma^{\top}, 1$, ZISP); Sablino (ca. $59.63^{\circ} \mathrm{N} 30.76^{\circ} \mathrm{E}$ ), $18-19$. VI.1923, A. Stackelberg (3 $0^{7} \mathrm{O}^{7}, 4$ 우, ZISP); Terioki [= Zelenogorsk] $\left(60.1954^{\circ} \mathrm{N} 29.6999^{\circ} \mathrm{E}\right)$, without data, Yu. Vagner $\left(1 \mathrm{O}^{\top}\right.$, 3 우, ZISP); Tolmachevo $\left(58.8565^{\circ} \mathrm{N} 29.8956^{\circ} \mathrm{E}\right), 2$. VII.1955, A. Stackelberg (1 $\circ$, ZISP); Magadan Oblast: Gizhiga $\left(61.9487^{\circ} \mathrm{N}\right.$ $\left.160.3772^{\circ} \mathrm{E}\right)$, 5.IX.1987, Gorodkov ( $10^{\top}, 1$ \%, ZISP); Madaun $\left(60.6063^{\circ} \mathrm{N} 150.6963^{\circ} \mathrm{E}\right), 2$. VII.1971, Gorodkov (3 $\sigma^{\top} \mathrm{O}^{\top}, 1$ 우, ZISP); Sokol $\left(59.9193^{\circ} \mathrm{N} 150.7527^{\circ} \mathrm{E}\right), 25 . \mathrm{VIII} .1966$, Gorodkov (1 O" ZISP); Chaybukha $\left(61.8016^{\circ} \mathrm{N} 160.4132^{\circ} \mathrm{E}\right)$, 1.IX.1987, Gorodkov 
(2 $\sigma^{\Upsilon} \sigma^{7}$, ZISP); Murmansk and Murmansk Oblast: Vud'yavr Lake Basin (ca. 67.6464 $\left.{ }^{\circ} \mathrm{N} 33.6449^{\circ} \mathrm{E}\right), 26$. VI.1930 and 13.VII.1935, Fridolin $\left(1 \sigma^{7}, 2\right.$ 우, ZISP); Laplandskiy Reserve $\left(67.6464^{\circ} \mathrm{N} 33.6449^{\circ} \mathrm{E}\right)$, 19.VII.-3.VIII.1975, Sychevskaya (1 $\sigma^{\top}, 2$ 웅, ZISP); Laplandskiy Reserve $\left(67.9268^{\circ} \mathrm{N} 32.0526^{\circ} \mathrm{E}\right), 10 . \mathrm{VII} .1976$, V. Sychevskaya (1 0 ZMUM); Lovozero $\left(68.005^{\circ} \mathrm{N} 35.0175^{\circ} \mathrm{E}\right)$, VIII.1933, Serebryanskiy $\left(1\right.$ ㅇ, ZISP); Murmansk env. $\left(68.9798^{\circ} \mathrm{N} 33.1148^{\circ} \mathrm{E}\right), 21 . \mathrm{VII} .2011, \mathrm{~A}$ Ozerov (1 $\left.\sigma^{7}, \mathrm{ZMUM}\right)$; Imandra station $\left(67.8502^{\circ} \mathrm{N} 33.2588^{\circ} \mathrm{E}\right)$, 9.VIII.1923, Frido-lin (1 $\sigma^{7}$, ZISP); Khibiny station $\left(67.6736^{\circ} \mathrm{N}\right.$ $33.2126^{\circ} \mathrm{E}$ ), 13-18.VIII.1928, Cheburova (4 oO, ZISP); Olen'ya station [= Olenegorsk] $\left(68.1365^{\circ} \mathrm{N} 33.2985^{\circ} \mathrm{E}\right), 15$. VIII.1923, V. Kuznetsov ( $1 \mathrm{O}^{\top}, 4$ 우, ZISP); Murmansk env. $\left(68.96^{\circ} \mathrm{N} 33.08^{\circ} \mathrm{E}\right), 8-10$. VII.1930, Cheburova (1 9 , ZISP); Tuva: Mondy env. $\left(51.6758^{\circ} \mathrm{N}\right.$ $\left.100.9925^{\circ} \mathrm{E}\right), 18-20$. VII.1965, Gorodkov (4 $\sigma^{\top} \sigma^{\top}, 1$ \%, ZISP); Il'chir Lake env. $\left(51.9762^{\circ} \mathrm{N} 100.9877^{\circ} \mathrm{E}\right), 31 . \mathrm{VII} .1965$, Gorodkov (1 $\sigma^{7}$, ZISP); Tyumen' Oblast: $\left(63.818^{\circ} \mathrm{N} 59.562^{\circ} \mathrm{E}\right), 1-4 . \mathrm{VII} .2010, \mathrm{~K}$ Tomkovich (1 $\left.\sigma^{7}, \mathrm{ZMUM}\right) ; 80 \mathrm{~km} \mathrm{~W}$ of Samburg (ca. $67.0357^{\circ} \mathrm{N} 76$. $\left.5411^{\circ} \mathrm{E}\right), 28$.VII.1977, Gorodkov (1 $\sigma^{7}$, ZISP); same place, 7-9.VIII. 1976, D. Kasparyan (2 $\sigma^{7} \sigma^{7}$, ZISP); the Sob' River Basin (ca. 66 $\left.9376^{\circ} \mathrm{N} 65.6706^{\circ} \mathrm{E}\right), 30$.VII.1925, Fridolin ( $2 \sigma^{7} \sigma^{7}, 1$ \%, ZISP); Labytnangi $\left(66.6579^{\circ} \mathrm{N} 66.3919^{\circ} \mathrm{E}\right), 24$. VII.1973, R. Kamenskaya (1 $\sigma^{7}, 1$ +, ZMUM); Labytnangi $\left(66.6611^{\circ} \mathrm{N} 66.3945^{\circ} \mathrm{E}\right), 14$.VIII.1985, Gorodkov (1 ऽ, ZISP); same place, 30.VI., 6-10.VII., 4-16.VIII.1973, 2 11.VII., 2 and 18 VIII.1974, 22.VIII.1978, Sychevskaya (15 ठ ठ $\sigma^{\top}, 8$ 우, ZISP); Labytnangi env. (66.6611 $\left.{ }^{\circ} \mathrm{N} 66.3945^{\circ} \mathrm{E}\right)$, VII.1971, Ol'shvang (5 $\sigma^{7} \sigma^{7}$, ZISP); Neroyka (ca. 64.57N 59.67E), $650 \mathrm{~m}$, 11.VI.1989, Malozemov (13 $\sigma^{7} \sigma^{7}, 5$ of , ZISP); the Khadyta River $\left(66.873^{\circ} \mathrm{N} 74.0574^{\circ} \mathrm{E}\right), 9 . \mathrm{VII} .1974$ and 8.VIII.1974, Bogacheva (3 $\sigma^{7} \sigma^{\top}, 1$ o, ZISP); Salekhard $\left(66.53^{\circ} \mathrm{N} 66.6135^{\circ} \mathrm{E}\right), 3 . \mathrm{VIII} .1961,20$ VIII.1972, Gorodkov (2 $\sigma^{7} \sigma^{7}$, ZISP); Yakutia: $144 \mathrm{~km}$ NW of Tiksi

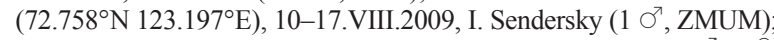
Aykhal $\left(65.945^{\circ} \mathrm{N} 111.4961^{\circ} \mathrm{E}\right), 19 . \mathrm{VIII} .1988$, Gorod-kov (1 ơ, 1 웅 ZISP); airport Saskylakh (71.9345 $\left.{ }^{\circ} \mathrm{N} 114.083^{\circ} \mathrm{E}\right), 24$.VII.1988, Gorodkov (5 $\sigma^{7} \sigma^{7}$, ZISP); Verkhoyansk $\left(67.5483^{\circ} \mathrm{N} 133.3961^{\circ} \mathrm{E}\right), 12 . \mathrm{VIII}$

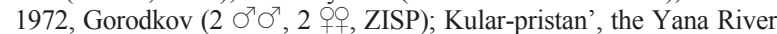
$\left(70.5665^{\circ} \mathrm{N} 134.9821^{\circ} \mathrm{E}\right), 2$. VIII.1988, Gorodkov (1 $\sigma^{\mathrm{T}}$, ZISP); Kyusyur $\left(70.6874^{\circ} \mathrm{N} 127.366^{\circ} \mathrm{E}\right), 13-19$. VII.1957, Gorodkov $\left(10 \bigcirc^{7} \sigma^{7}, 9\right.$ OO, ZISP); Nel'kan $\left(57.6548^{\circ} \mathrm{N} 136.163^{\circ} \mathrm{E}\right), 1-2$.VIII.1903, Popov (1 $\sigma^{\top}$, ZISP); Sevast'yan Lake, $12 \mathrm{~km} \mathrm{~S}$ of Tiksi $\left(71.5377^{\circ} \mathrm{N} 128.8569^{\circ} \mathrm{E}\right)$, 18.VII.1957, Kapitonov (1 9 , ZISP); Stolb I. $\left(72.3956^{\circ} \mathrm{N} 126.6588^{\circ} \mathrm{E}\right)$ the Lena delta, 25.VII.1957, Gorodkov (1 $\sigma^{7}$, ZISP); Teplyy Klyuch $\left(62.7836^{\circ} \mathrm{N} 136.8096^{\circ} \mathrm{E}\right), 21 . \mathrm{VIII} .1974$, Gorod-kov (1 O', ZISP); Tiksi $\left(71.6351^{\circ} \mathrm{N} 128.8579^{\circ} \mathrm{E}\right), 2-9$.VII. and $11-17$.VIII.1957, Gorodkov

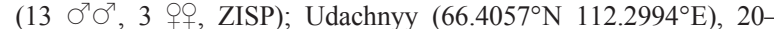
21.VII.1988, Gorodkov (4 $\sigma^{\top} \sigma^{\top}, 2$ 우, ZISP); Uryung-Khaya $\left(72.8124^{\circ} \mathrm{N} 113.2316^{\circ} \mathrm{E}\right), 7$. VIII.1988, Gorodkov (1 ㅇ, ZISP); ChayTumus $\left(72.3166^{\circ} \mathrm{N} 125.7333^{\circ} \mathrm{E}\right), 25 . \mathrm{VII} .1957$, Gorodkov $\left(2 \sigma^{7} \sigma^{7}, 4\right.$ of, ZISP); Chekurovka (71.0469 $\left.{ }^{\circ} \mathrm{N} 127.5255^{\circ} \mathrm{E}\right), 24$.VII.1957, Gorod-

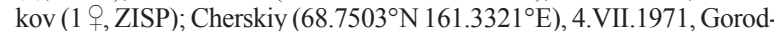

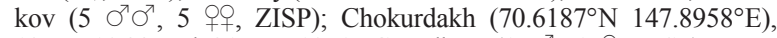
13.VII.1966 and 21.VIII.1971, Gorodkov (1 o', 1 +, ZISP); same place, 19.VII.1971, Chelnokov (1 $\sigma^{7}$, ZISP); Ebelyakh, the Anabar River $\left(70.8849^{\circ} \mathrm{N} 113.5704^{\circ} \mathrm{E}\right), 22$.VII.1988, Gorodkov (1 9 , ZISP).

DESCRIPTION. Male, female. Small or mediumsized flies (3.6-5.6 mm long).

Head. Frontal vitta reddish-yellow, matt; frontoorbital plate black in upper half and reddish-yellow in lower half, greyish dusted. Ocellar triangle black, with grey pollen. Face and parafacial black in male, completely white in female. Gena and postgena usually black in upper half and yellow in lower half in male, completely white in female. Postcranium black, greyish dusted, covered with black setae in upper third and white hairs in lower part. 3 orbitals, 2-3 frontals, 1 ocellar, 1 small postocellar, 1 inner vertical, 1 outer vertical; 2-3 pairs of vibrissae present. Scape and pedicel from brown to black. Postpedicel black, rounded apically, approximately 2 times as long as wide. Arista black, basally thickened, bare. Clypeus and proboscis black. Palpus broadened towards apex, yellow.
Thorax and scutellum black, greyish dusted. Acrostical hairs in two rows, dorsocentrals $(4-5)+(3-4)$, intraalars $1+2$, supra-alars $1+2$, postpronotals 2 , notopleurals 2 , postalars 2. Proepisternum with hairs in central part, with 1-2 long pale setae near lower margin. Proepimeron with one pale or black seta and several small hairs. Anepisternum covered with pale hairs in posterior half, with 3-5 black setae along posterior margin. Katepisternum covered with pale hairs in posterior half and 1 strong seta in upper posterior corner. Anepimeron bare. Scutellum black, greyish dusted, with a pair of strong basal scutellar and a pair of strong apical scutellar setae.

Legs yellow, only mid and hind coxae black. Fore femur with a row of posterodorsal setae, with numerous hairs anteriorly and ventrally. Fore tibia with short black ventral spinules in two rows on whole length, with 1 dorsal seta near middle, with 1 preapical dorsal seta and usually with 1 apical posterior seta. Mid femur with irregular row of anterior setae, with 1 preapical posterior and 1 preapical posterodorsal setae. Mid tibia with 1 anterodorsal seta near middle, and a ring of apicals. Hind femur with a row of anterodorsal setae. Hind tibia with 2 anterodorsal, 1-2 posterodorsal and 1 preapical dorsal setae, also with 1 apical anterodorsal and 1 apical anteroventral setae.

Wing slightly darkened; veins brownish. $\mathrm{R}_{1}$ bare dorsally. Calypters, margins of calypters, and halteres yellowish.

Abdomen black, greyish dusted; female abdominal tergites 6-8 reddish. Male sternite 4 rectangular, slightly wider than long (Fig. 14). Male sternite 5 symmetrical; lobes simple, narrow, sharpen apically (Fig. 28). Epandrium and surstyli as in Figs 45, 46. Female sternites 5-7 as in Fig. 69; sternite 5 rectangular, not wider than sternite 6 and sternite 7; sternite 7 ovoid, wider than long.

DISTRIBUTION. Russia (Fig. 81): Arkhangelsk Oblast, Buryatia, Chukotka, Irkutsk Oblast, Kamchatka Kray, Karelia, Komi, Krasnoyarsk Kray, Leningrad Oblast, Magadan Oblast, Murmansk Oblast, Tuva, Tyumen' Oblast, Yakutia. - Europe, North America.

Microprosopa (Microprosopa) heteromyzina (Zetterstedt, 1838)

Figs $15,29,47,48,70,82$.

heteromyzina Zetterstedt, 1838: 723 (Scatomyza). Type-locality: "Lapponia Umensi... Lycksele" (Sweden).

Noted by Ozerov \& Krivosheina [2014: 213] from Far East. Reporting of the species for Siberia (Krasnoyarsk Kray) [Ozerov \& Barkalov, 2014: 562] is incorrect, both specimens proved to be $M$. haemorrhoidalis.

MATERIAL EXAMINED. Altay: Seminskiy pass, $1252 \mathrm{~m}$ $\left(51.05^{\circ} \mathrm{N} 85.62^{\circ} \mathrm{E}\right), 30 . \mathrm{VI} .2009$, A. Barkalov $\left(2 \sigma^{\top} \sigma^{\top}\right.$, ISEA and ZMUM); Seminsky pass, the Sarlyk River (51.11 $\left.{ }^{\circ} \mathrm{N} 85.60^{\circ} \mathrm{E}\right), 1200$ m, 28-30.VI.2016, N. Vikhrev (3 $\sigma^{7} \sigma^{7}$, ZMUM); Karelia: Paanayarvi $\left(66.1625^{\circ} \mathrm{N} 30.5436^{\circ} \mathrm{E}\right)$, 1.VII.1998, Polevoy (1 $\sigma^{\top}$, ZMUM).

ADDITIONAL MATERIAL EXAMINED. Holotype + of $\mathrm{Mi}$ croprosopa heteromyzina (Zetterstedt), with labells: 1) small black tag, 2) “S. heteromyzina + Lycks", 3)"Holotypus Scatomyza heteromyzina Zett. Labelled 1954 by J.R. Vockeroth" (MZLU).

DESCRIPTION. Male, female. Medium-sized flies (4.2-5.3 mm long). 

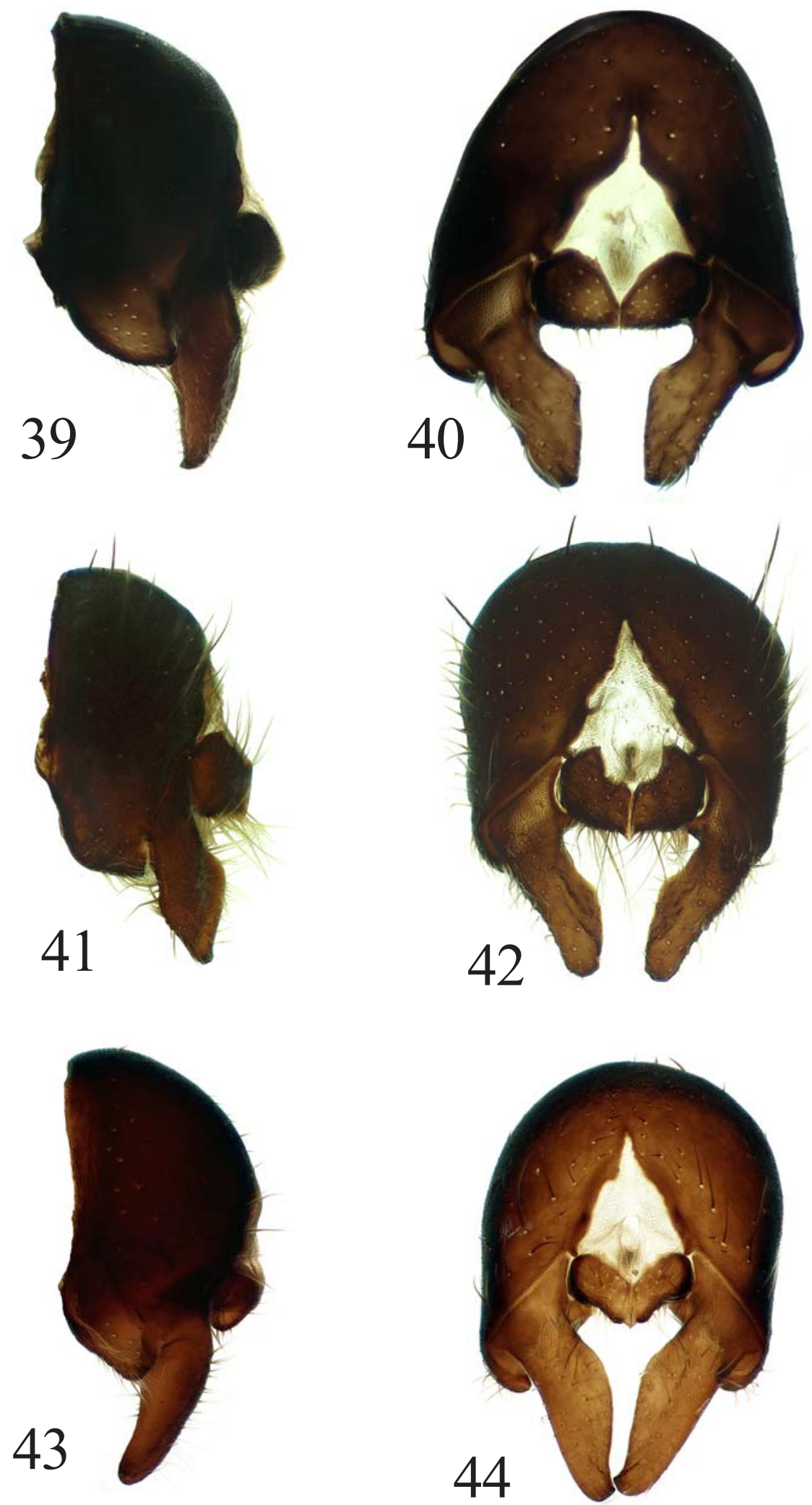

Figs 39-44. Epandrium and surstyli of Microprosopa: $39-$ M. albipennis (Zetterstedt), lateral view; 40 — same, dorsal view; 41 M. crinipes Ringdahl; 42 - same, dorsal view; 43 - M. frigida (Holmgren), lateral view; 44 - same, dorsal view.

Рис. 39-44. Эпандрий и сурстили Microprosopa: 39 - M. albipennis (Zetterstedt), сбоку; 40 - то же, сверху; 41 - M. crinipes Ringdahl, сбоку; 42 - то же, сверху; 43 - M. frigida (Holmgren), сбоку; 44 — то же, сверху. 

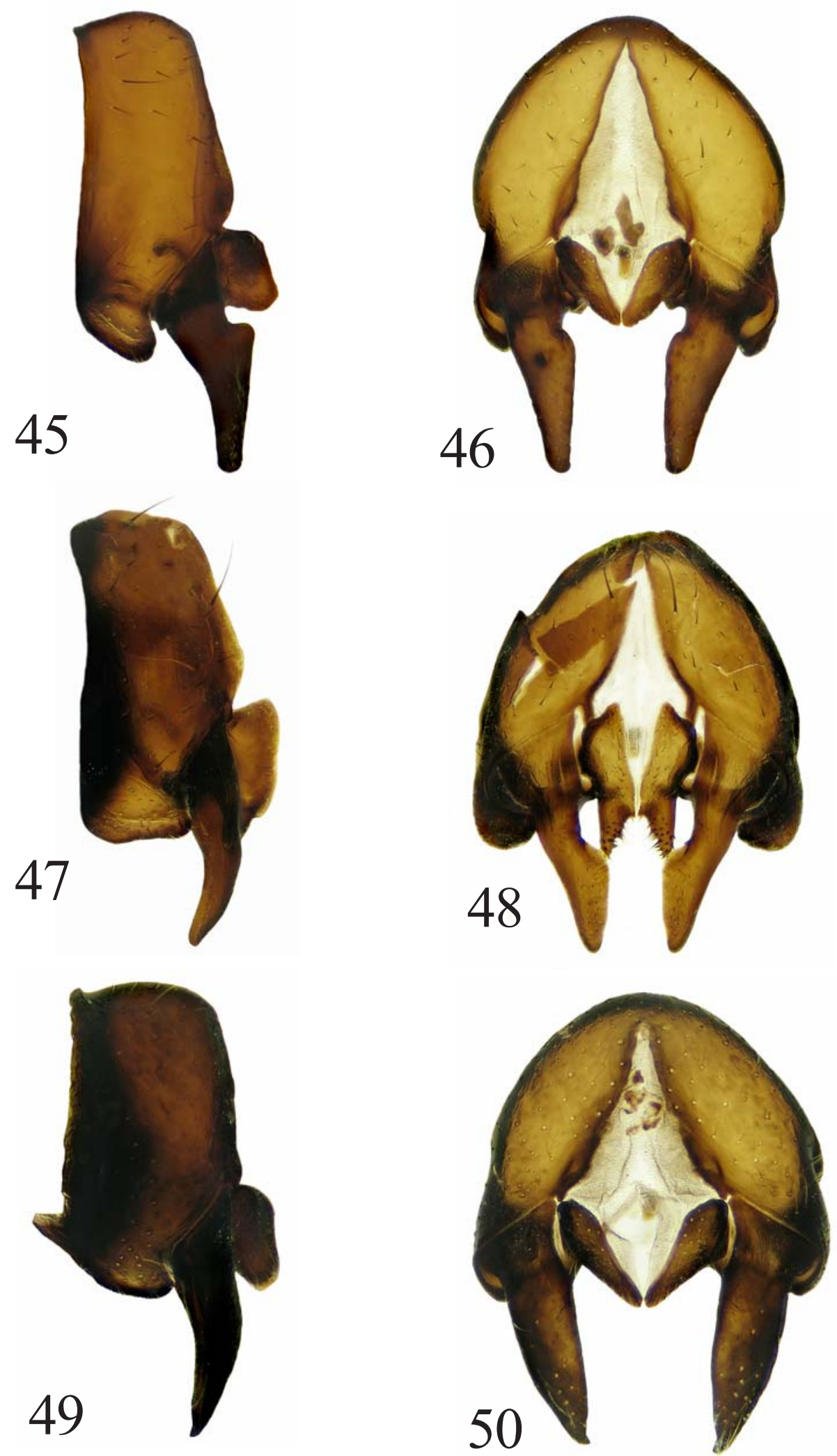

Figs 45-50. Epandrium and surstyli of Microprosopa: 45 - M haemorrhoidalis (Meigen), lateral view; 46 - same, dorsal view; 47 - M. heteromyzina (Zetterstedt); 48 - same, dorsal view; 49 - M. lineata (Zetterstedt), lateral view; 50 - same, dorsal view.

Рис. 45-50. Эпандрий и сурстили Microprosopa: $45-$ - M. haemorrhoidalis (Meigen), сбоку; 46 — то же, сверху; $47-M$. heteromyzina (Zetterstedt), сбоку; 48 — то же, сверху; 49 - M. lineata (Zetterstedt), сбоку; 50 - то же, сверху. 

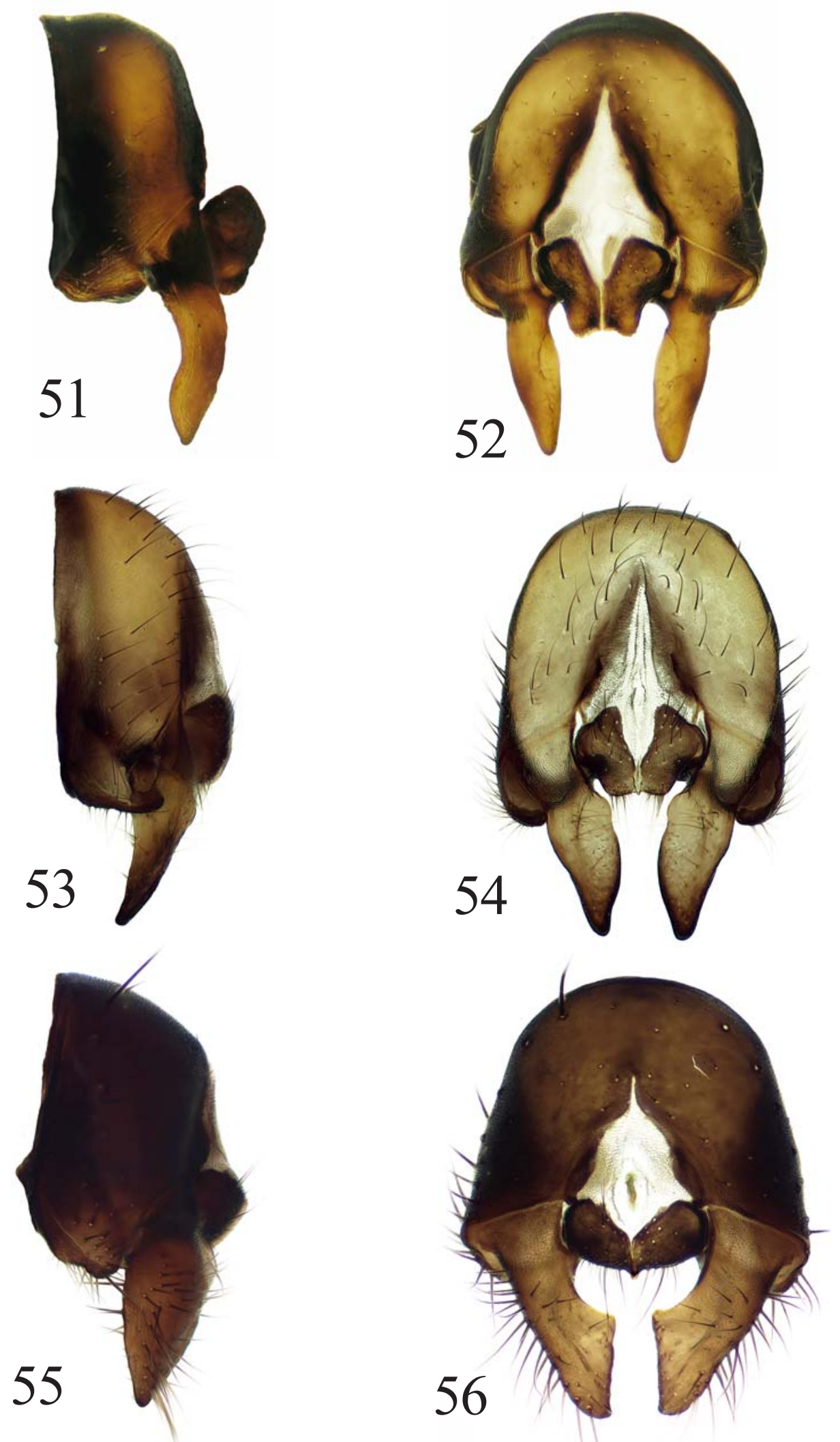

Figs 51-56. Epandrium and surstyli of Microprosopa: $51-M$. pallidicauda (Zetterstedt), lateral view; 52 - same, dorsal view; 53 - M. paveli Ozerov et Krivosheina; 54 - same, dorsal view; 55 - M. portenkoi Stackelberg, lateral view; 56 - same, dorsal view.

Рис. 51-56. Эпандрий и сурстили Microprosopa: 51 - M. pallidicauda (Zetterstedt), сбоку; 52 — то же, сверху; 53 - M. paveli Ozerov et Krivosheina, сбоку; 54 - то же, сверху; 55 - M. portenkoi Stackelberg, сбоку; 56 - то же, сверху. 

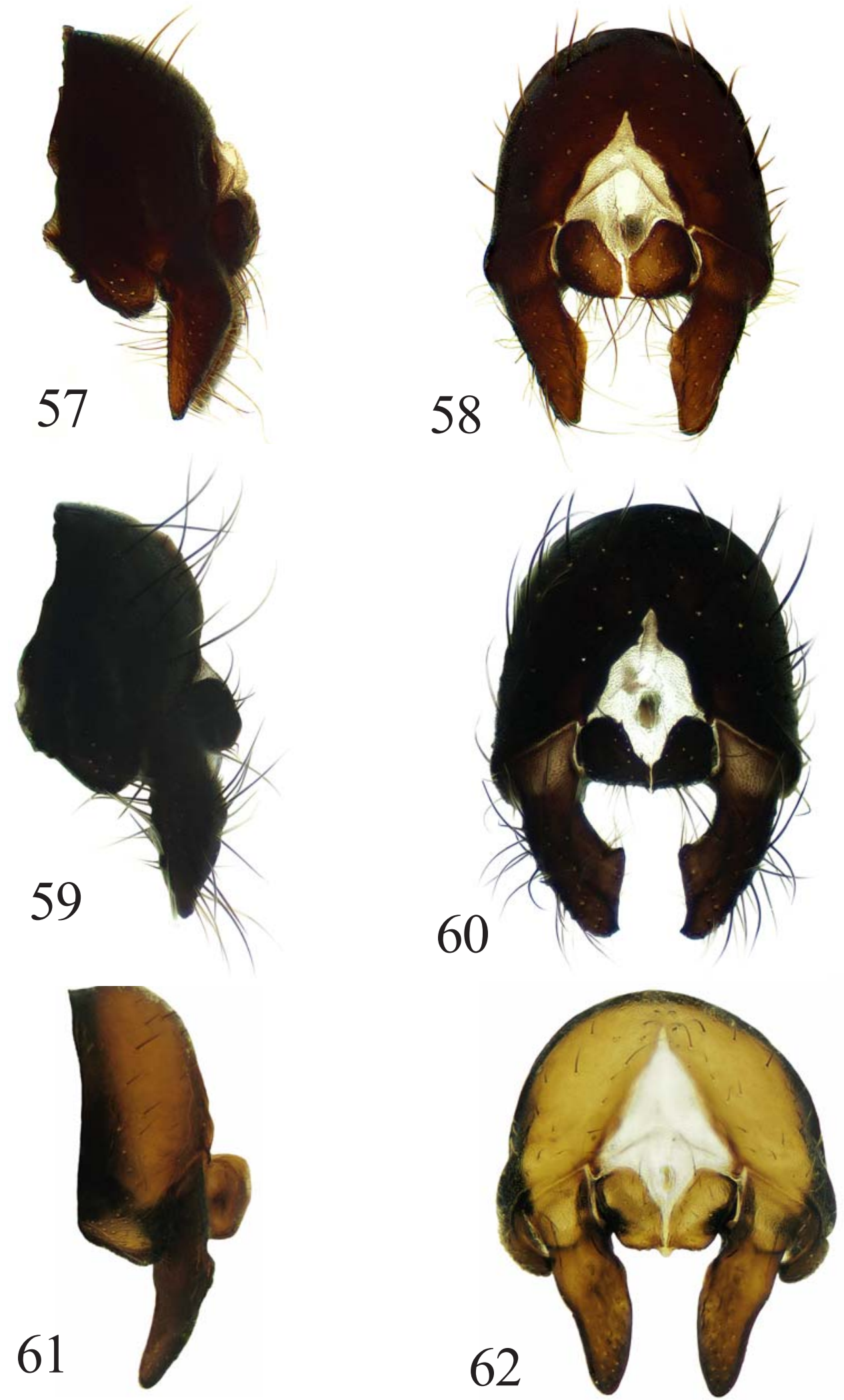

Figs 57-62. Epandrium and surstyli of Microprosopa: $57-M$. taimyrica sp.n., lateral view; 58 - same, dorsal view; $59-M$. unguiculata (Malloch), lateral view; 60 - same, dorsal view; $61-$ M. zlobini Ozerov, lateral view; 62 - same, dorsal view.

Рис. 57-62. Эпандрий и сурстили Microprosopa: 57 - M. taimyrica sp.n., сбоку; 58 - то же, сверху; 59 - M. unguiculata (Malloch), сбоку; 60 - то же, сверху; 61 - M. zlobini Ozerov, сбоку; 62 — то же, сверху. 
Head. Frontal vitta reddish-yellow completely or blackish in upper half, matt; fronto-orbital plate black, greyish dusted. Ocellar triangle black, with grey pollen. Face and parafacial from black to yellow in male, completely pale yellow in female. Gena and postgena pale yellow. Postcranium black in upper part and yellow in lower quarter, greyish dusted, covered with black setae in upper third and white hairs in lower part. 3 orbitals, 2-3 frontals, 1 ocellar, 1 small postocellar, 1 inner vertical, 1 outer vertical; usually 2 pairs of pale vibrissae present. Scape and pedicel from brown to black. Postpedicel black, rounded apically, approximately 2 times as long as wide. Arista black, basally thickened, bare. Clypeus and proboscis black. Palpus broadened towards apex, yellow.

Thorax and scutellum black, greyish dusted. Acrostical hairs in two rows, dorsocentrals $3+3$, intra-alars $1+2$, supra-alars $1+2$, postpronotals 2 , notopleurals 2 , postalars 2. Proepisternum with hairs in central part, with 1-2 long pale setae near lower margin. Proepimeron with one pale or black seta. Anepisternum covered with pale hairs in posterior half, with 3 black setae along posterior margin. Katepisternum covered with pale hairs in posterior half and 1 strong seta in upper posterior corner. Anepimeron bare. Scutellum black, greyish dusted, with a pair of strong basal scutellar and a pair of strong apical scutellar setae.

Legs yellow, only mid and hind coxae brown or black. Fore femur with irregular row of posterodorsal setae, with numerous hairs anteriorly and ventrally. Fore tibia with short black ventral spinules in two rows on whole length, with 1 dorsal seta near middle, with 1 preapical dorsal seta and usually with 1 apical posterior seta. Mid femur with a row of anterior setae, with 1 preapical posterior and 1 preapical posterodorsal setae. Mid tibia with 1 anterodorsal and 1 posterodorsal setae near middle, and a ring of apicals. Hind femur with a row of anterodorsal setae. Hind tibia with 1-2 anterodorsal, 1-2 posterodorsal and 1 preapical dorsal setae, also with 1 apical anterodorsal and 1 apical anteroventral setae.

Wing slightly darkened; veins brownish. $\mathrm{R}_{1}$ bare dorsally. Calypters, margins of calypters, and halteres yellowish.

Abdomen black in male and usually yellow in female, greyish dusted. Male sternite 4 rectangular, about 2 times as wide as long (Fig. 15). Male sternite 5 symmetrical; lobes simple, wide, rounded apically (Fig. 29). Epandrium and surstyli as in Figs 47, 48. Female sternites $5-7$ as in Fig. 70; lateral margins of sternite 7 fused with lateral margins of sternite 7 forming syntergosternite; sternite 7 as long as sternite 5 .

DISTRIBUTION. Russia (Fig. 82): Altay, Karelia. - Europe, North America.

Microprosopa (Microprosopa) lineata (Zetterstedt, 1838)

Figs 3, 16, 30, 49, 50, 71, 83 .

lineata Zetterstedt, 1838: 732 (Cordylura). Type-locality: "Lapponia Tornensi... ad Juckasjervi..." (Sweden, Jukkasjärvi).

The species was recorded for Russia by Gorodkov [1986: 24] from the European part without indicating a specific locality, also from Arkhangelsk Oblast [Engelmark, 1999: 159] and Krasnoyarsk Kray (Taymyr) [Ozerov \& Barkalov, 2014: 562].

MATERIAL EXAMINED. Arkhangelsk Oblast: Nar'yan-Mar $\left(67.6370^{\circ} \mathrm{N} 52.0214^{\circ} \mathrm{E}, 67.6317^{\circ} \mathrm{N} 52.9857^{\circ} \mathrm{E}\right), 6-13 . \mathrm{VII} .2008$, N.E. Vikhrev, A.L. Ozerov (7 $\sigma^{7} \sigma^{7}, 10$ 우, ZMUM); Chukotka: bank of the Anadyr River (64.72 $\left.{ }^{\circ} \mathrm{N} 175.21^{\circ} \mathrm{E}\right), 25$.VI.-19.VII.2014, A. Barkalov (1 $0^{7}$, ISEA); the lower reaches of the Anadyr' River $\left(64.83^{\circ} \mathrm{N}, 175.96^{\circ} \mathrm{E}\right)$; Komi: Vorkuta $\left(67.5^{\circ} \mathrm{N} 64.0^{\circ} \mathrm{E}\right), 19-25$.VII. 2010, N. Vikhrev (2 우, ZMUM); Krasnoyarsk Kray: Agapa (ca. $\left.71.412^{\circ} \mathrm{N} 89.2689^{\circ} \mathrm{E}\right)$, the Pyasina River, 16-19.VII.1967, Gorod$\operatorname{kov}\left(80^{7} \sigma^{7}, 8\right.$ 우, ZISP); Dudinka $\left(69.4^{\circ} \mathrm{N} 86.2^{\circ} \mathrm{E}\right), 26-29$. VII. 2011, N. Vikhrev (1 +, ZMUM); Glubokoe Lake, $60 \mathrm{~km}$ O of Noril'sk (ca. $69.373^{\circ} \mathrm{N} 89.799^{\circ} \mathrm{E}$ ), 8.VII.1967, Gorodkov (1 $0^{7}$, ZISP); Murmansk Oblast: the Kola River $\left(68.8869^{\circ} \mathrm{N} 33.0236^{\circ} \mathrm{E}\right), 19$.VII.2011, A. Ozerov (2 $0^{7} 0^{7}, 2$ 우, ZMUM); Tyumen' Oblast: $85 \mathrm{~km}$ WSW of Antipayuta (ca. $68.8208^{\circ} \mathrm{N} 75.3442^{\circ} \mathrm{E}$ ), 31.VII.1977, Gorodkov (1 $\mathrm{O}^{7}, 1$, , ZISP); Yakutia: the Atyrayyana River near Zhigansk $\left(66.7635^{\circ} \mathrm{N} 123.329^{\circ} \mathrm{E}\right), 4$.VII.1875, Chekanovskiy (1 + , ZISP).

DESCRIPTION. Male, female. Small or mediumsized flies (4.0-5.7 mm long). Almost all setae on head, thorax and legs pale.

Head. Frontal vitta mostly reddish-yellow, only in upper third darkened, matt; fronto-orbital plate and ocellar triangle black, greyish dusted. Face, parafacial, gena and postgena pale yellow. Postcranium black, greyish dusted, covered with hairs. 3 orbitals, 2-3 frontals, 1 ocellar, 1 small postocellar, 1 inner vertical, 1 outer vertical; 2 pairs of vibrissae present. Antenna black, but pedicel sometimes brownish. Postpedicel rounded apically, approximately 2 times as long as wide. Arista black, basally thickened, bare. Clypeus and proboscis black. Palpus broadened towards apex, yellow.

Thorax and scutellum black, greyish dusted. Scutum with two shining narrow stripes medially and fore shining spots (Fig. 3). Acrostical hairs in two rows, dorsocentrals $3+3$, intra-alars $1+2$, supra-alars $1+2$, postpronotals 2, notopleurals 2, postalars 2. Proepisternum with hairs in central part, with 1-2 setulae near lower margin. Proepimeron with one setula. Anepisternum covered with hairs in posterior half, with 2-4 setae along posterior margin. Katepisternum covered with hairs in posterior half and 1 strong seta in upper posterior corner. Anepimeron bare. Scutellum black, greyish dusted, with a pair of basal scutellar and a pair of apical scutellar setae.

Fore coxa brown or blackish, mid and hind coxae black; femora, tibiae and tarsi of all legs yellow. Fore femur with rows of posterodorsal and posteroventral setae, with numerous hairs posteriorly and posteroventrally. Fore tibia with short black spinules ventrally in two irregular rows on whole length, with 1 dorsal seta near middle, with 1 preapical dorsal and apical posterior setae. Mid femur with irregular row of anterior setae, with 1 preapical posterior and 1 preapical posterodorsal setae, also with pale hairs ventrally. Mid tibia with 1 anterodorsal and 1 posterodorsal setae near middle, also with a ring of apicals. Hind femur with a rows of anterodorsal and anteroventral setae, also with numerous hairs posteriorly and posteroventrally. Hind tibia with 2-3 anterodorsal, 12 posterodorsal, 1 preapical dorsal, and $0-1$ preapical posteroventral setae, also with 1 apical anteroventral seta. 

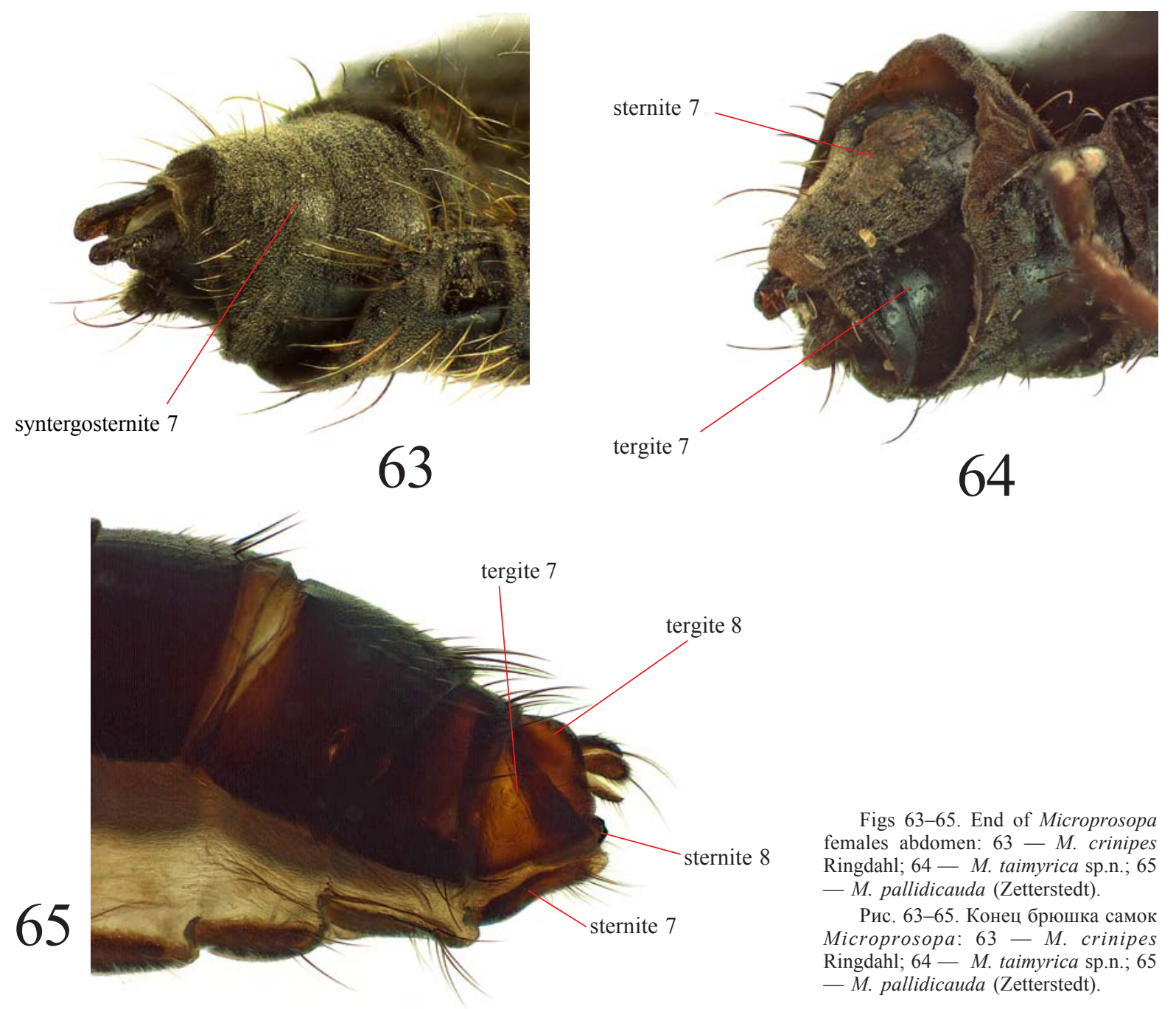

Figs 63-65. End of Microprosopa females abdomen: $63-$ M. crinipes Ringdahl; $64-$ M. taimyrica sp.n.; 65 - M. pallidicauda (Zetterstedt).

Рис. 63-65. Конец брюшка самок Microprosopa: $63-$ M. crinipes Ringdahl; $64-$ M. taimyrica sp.n.; 65 - M. pallidicauda (Zetterstedt).

Wing slightly darkened; veins blackish. $\mathrm{R}_{1}$ bare dorsally. Calypters, margins of calypters, and halteres whitish or yellowish.

Abdomen black, greyish dusted, sometimes male tergites 3-6 shining along lateral margins. Male sternite 4 trapezoid, slightly wider than long (Fig. 16). Male sternite 5 symmetrical; lobes simple, narrow (Fig. 30). Epandrium and surstyli as in Figs 49, 50. Female sternites 5-7 as in Fig. 71; sternite 5 rectangular, not wider than sternite 6 and sternite7; sternite 7 ovoid, as long as wide.

DISTRIBUTION. Russia (Fig. 83): Arkhangelsk Oblast, Chukotka, Komi, Krasnoyarsk Kray, Murmansk Oblast, Tyumen' Oblast, Yakutia. - Europe, North America.

Microprosopa (Microprosopa) pallidicauda (Zetterstedt, 1838)

Figs 1, 4, 9, 17, 31, 51, 52, 65, 72, 84 .

pallidicauda Zetterstedt, 1838: 733 (Cordylura). Type-locality: "Lycksele Lapponiae Umensis ... Juckasjervi Lapponiae Tornensis... (Lapponia Suecica et sylvatica)" (Sweden). melanura Zetterstedt, 1838: 731 (Cordylura). Type-locality: "Kengis Lapponiae Tornensis... Stensele, Umenaes, Wilhelmina et Åsele, Lapponia Umensis" (Sweden) — syn.n.'

pallicauda, error

C. melanura was described from an unspecified number of both sexes from "Lycksele Lapponiae Umensis ... Juckasjervi Lapponiae Tornensis... (Lapponia Suecica et sylvatica)", Sweden. Later Zetterstedt suppored this species to be identical to Cordylura pallipes Zetterstedt, 1838 [Zetterstedt, 1846: 1993, footnote] and marked these specimens in such way (see below). Becker [1905: 18] synonymized this species with Microprosopa haemorrhoidalis Meigen. Gorodkov mentioned this species as the synonym of M. haemorrhoidalis [Gorodkov, 1986: 25] at the same time as the synonym of Okeniella caudata (Zetterstedt, 1838) [Gorodkov, 1986: 22].

2 syntypes of C. melanura were discovered in Zetterstedt's collection in Lund. One of them labelled 1) small black tag, 2) " $C$. pallipes $\sigma^{7}$. melanura olim", 3)"Lectotypus Cordylura melanura Zett. Designated by Vockeroth 19"[not published designation], 4)"Microprosopa pallidicauda Zett. Det. J.R. Vockeroth 1963" was studied by me, designated it herewith and labelled it as lectotype of Cordylura melanura. It is conspecific with Microprosopa pallidicauda (Zetterstedt, 1838). The second specimen has label "C. caudatum 9 (C. melanura Ins. L.) Lycksele" [personal comm. by Rune Bygebjerg], not studied. 
Noted for Russia from Kola Peninsula and Arkhangelsk Oblast [Hackman, 1956: 58; Humala \& Polevoi, 2009: 72], Siberia [Gorodkov, 1986: 25 (without indicating a specific locality)], and Far East [Ozerov \& Krivosheina, 2014: 214].

MATERIAL EXAMINED. Altay: Kosh-Agach env. $\left(50.01^{\circ} \mathrm{N}\right.$ $\left.88.66^{\circ} \mathrm{E}\right), 1750 \mathrm{~m}, 2-4$. VII.2016, N. Vikhrev (1 9 , ZMUM); Seminsky pass (51.06N 85.59E), $1650 \mathrm{~m}, 27-30 . V I .2016$, N. Vikhrev (10 $\bigcirc^{\top} \sigma^{\top}, 4$ Oᄋ, ZMUM); Seminsky pass, the Turala River $\left(50.99^{\circ} \mathrm{N}\right.$ $\left.85.68^{\circ} \mathrm{E}\right), 1350 \mathrm{~m}, 8-12$.VII.2016, N. Vikhrev (1 $\sigma^{7}$, ZMUM) Arkhangelsk Oblast: Khal'mer'yu (ca. $\left.67.5507^{\circ} \mathrm{N} 53.962^{\circ} \mathrm{E}\right), 18$.VII 1962, Gorodkov (1 ${ }^{7}$, ZISP); $70 \mathrm{~km} \mathrm{~N}$ of Nar'yan-Mar (ca. 68

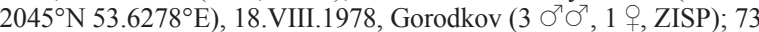
km NW of Nar'yan-Mar (ca. 67.9814N 51.6083E), 3.VIII.1978, Gorodkov (7 O $^{7} \mathrm{O}^{7}, 1$ ㅇ, ZISP); Nar'yan-Mar $\left(67.6271^{\circ} \mathrm{N} 53.0003^{\circ} \mathrm{E}\right.$ $\left.67.6317^{\circ} \mathrm{N} 52.9857^{\circ} \mathrm{E}, 67.6344^{\circ} \mathrm{N} 52.99721^{\circ} \mathrm{E}\right), 7-13$.VII.2008, N.E. Vikhrev, A.L. Ozerov (24 $\sigma^{\top} \sigma^{\top}, 11$ 우, ZMUM); Nar’yan-Mar $\left(67.6369^{\circ} \mathrm{N} 53.0316^{\circ} \mathrm{E}\right), 2$ and 20.VIII.1978, Gorodkov (5 $\sigma^{\top} \sigma^{7}, 2$ 0 , ZISP); Nenetskiy Reserve, Cordon "Bol'shoy Gusinets" (68. $\left.1759^{\circ} \mathrm{N} 53.6453^{\circ} \mathrm{E}\right), 9-10 . \mathrm{VII} .2008$, A.L. Ozerov (3 $\sigma^{7} \sigma^{7}, 3$ 우, ZMUM); Nizhnyaya Pesha (66.7518 $\left.{ }^{\circ} \mathrm{N} 47.7605^{\circ} \mathrm{E}\right), 22-23 . \mathrm{VIII} .1978$, Gorodkov ( $3 \sigma^{7} \sigma^{7}$, ZISP); the lower reaches of the Pechora River, Kashin I. (68.242 ${ }^{\circ}$ N 53.8567 $\left.{ }^{\circ} \mathrm{E}\right), 10 . \mathrm{VII} .2008$, A.L. Ozerov (3 $\sigma^{7} \sigma^{7}$ ZMUM); Nar'yan-Mar env. (67.6428 $\left.{ }^{\circ} \mathrm{N} 53.1963^{\circ} \mathrm{E}\right), 6 . \mathrm{VII} .2008$, A.L. Ozerov (1 $\left.\bigcirc^{7}, \mathrm{ZMUM}\right)$; Solovetskie I. $\left(65.0805^{\circ} \mathrm{N} 35.6863^{\circ} \mathrm{E}\right)$, 15 and 28.VI.1925, Birulya (3 $\sigma^{\top} \sigma^{\top}, 2$ 우, ZISP); Solovetskie I., Rebolda $\left(65.144^{\circ} \mathrm{N} 35.8282^{\circ} \mathrm{E}\right), 26 . V I .1896$, Birulya (1 9 , ZISP) Buryatia: Barguzinskiy Reserve (ca. $54.35^{\circ} \mathrm{N} 109.51^{\circ} \mathrm{E}$ ), 23 and 24.VII.1965, Negrobov ( $20^{7} \sigma^{7}, 1$ 9 , ZISP); Mondy env. $\left(51.6758^{\circ} \mathrm{N}\right.$ $100.9925^{\circ} \mathrm{E}$ ), 22 and 23.VII.1965, Gorodkov (1 $\sigma^{\top}, 2$ 우, ZISP); Kamchatka Kray: Apuka env. $\left(60.4437^{\circ} \mathrm{N} 169.6022^{\circ} \mathrm{E}\right), 1-$ 2.VIII.1959, Gorodkov (2 $\sigma^{7} \mathrm{O}^{7}$, ZISP); Valley of Geysers (ca. $54.43^{\circ} \mathrm{N}$ $\left.160.15^{\circ} \mathrm{E}\right), 30 . \mathrm{VII}$. and 12.VIII.1985, V. Zlobin (1 ๑ , 2 우, ZISP); Karelia: Poyakonda $\left(66.5898^{\circ} \mathrm{N} 33.8210^{\circ} \mathrm{E}\right)$, 8.VII.2010, A.L. Ozerov ( $8 \sigma^{7} \sigma^{7}, 3$ 오, ZMUM); Komi: Seida $\left(67.05^{\circ} \mathrm{N} 63.09^{\circ} \mathrm{E}\right)$ 23.VII.2010, N. Vikhrev (1 $\sigma^{7}$, ZMUM); Vil'gort $\left(60.5606^{\circ} \mathrm{N} 56\right.$ $\left.4652^{\circ} \mathrm{E}\right), 11 . \mathrm{VII} .1957$, Gabova $\left(2 \mathrm{\sigma}^{7} \mathrm{O}^{\top}\right.$, ZISP); Vorkuta $\left(67.5^{\circ} \mathrm{N}\right.$ 64.0 $\left.{ }^{\circ} \mathrm{E}\right), 19-25$. VII.2010, N. Vikhrev (6 $\sigma^{7} \sigma^{7}, 1$ ㅇ, ZMUM); Shchel'yabozh $\left(66.29^{\circ} \mathrm{N} 56.45^{\circ} \mathrm{E}\right), 15$.VIII.1978, Gorodkov $\left(4 \bigcirc^{7} \sigma^{7}\right.$ ZISP); Shchel'yayur $\left(65.33^{\circ} \mathrm{N} 53.43^{\circ} \mathrm{E}\right), 12$.VIII.1978, Gorodkov (1 $\sigma^{7}$, ZISP); Krasnoyarsk Kray: 104 km NNW of Noril'sk, the Nizhnyaya Agapa River (ca. $\left.70.0972^{\circ} \mathrm{N} 86.6883^{\circ} \mathrm{E}\right), 16 . \mathrm{VII} .1973$, Sukacheva, Zherikhin $\left(1 \Im^{\top}\right.$, ZISP); Turuchansk $\left(65.7972^{\circ} \mathrm{N} 87.9586^{\circ} \mathrm{E}\right)$, 28.VI.1967, Gorodkov (2 $\sigma^{7} \sigma^{7}$, ZISP); Dudinka $\left(69.4042^{\circ} \mathrm{N}\right.$ $\left.86.1822^{\circ} \mathrm{E}\right), 3$.VII.1967, Gorodkov (3 $\sigma^{\top} \sigma^{\top}$, ZISP); Igarka $\left(67.457^{\circ} \mathrm{N}\right.$ $86.598^{\circ} \mathrm{E}$ ), the Enisey River, 30.VI.1967, Gorodkov (6 $0^{7} \sigma^{7}, 1$, ZISP); Glubokoe Lake, $60 \mathrm{~km}$ E of Noril'sk (ca. $69.373^{\circ} \mathrm{N} 89.799^{\circ} \mathrm{E}$ ), 8.VII.1967, Gorodkov ( $\sigma^{7}, 1$, ZISP); Leningrad Oblast: Tolmachevo $\left(58.8565^{\circ} \mathrm{N} 29.8956^{\circ} \mathrm{E}\right), 2$.VII.1955, A. Stackelberg (1 웅 ZISP); Magadan Oblast: Koni Peninsula, Ploskiy Cape (59. $\left.1612798^{\circ} \mathrm{N} 151.6436005^{\circ} \mathrm{E}\right), 16 . \mathrm{VII} .2015$, N. Tridrikh $(1$ o, collection of Magadanskiy Reserve); Murmansk and Murmansk Oblast: Teriberka $\left(69.1641^{\circ} \mathrm{N} 35.1403^{\circ} \mathrm{E}\right), 3 . \mathrm{VII} .2008$, V. Semenov $\left(2 \sigma^{\top}, 1\right.$ $\circ$. ZMUM); Murmansk env. (68.96 $\left.{ }^{\circ} \mathrm{N} 33.08^{\circ} \mathrm{E}\right), 15$.VIII.1923, Fridolin (1 $\circ$, ZISP); Sakhalin Oblast: Iturup I., Rybaki $\left(45.2093^{\circ} \mathrm{N}\right.$ $\left.147.8519^{\circ} \mathrm{E}\right), 22 . V I .1968$, V. Richter (2 $\sigma^{\top} \sigma^{\top}$, ZISP); Tuva: the upper reaches of the Mogun-Buren' River (ca. $50.0419^{\circ} \mathrm{N} 89.8724^{\circ} \mathrm{E}$ ), 25.VII.1964, Narchuk (2 $\bigcirc^{7} \sigma^{7}$, ZISP); Tyumen' Oblast: $\left(63.766^{\circ} \mathrm{N}\right.$ $\left.59.716^{\circ} \mathrm{E}\right), 1-4$. VII.2010, K. Tomkovich (1 +, ZMUM); $\left(63.818^{\circ} \mathrm{N}\right.$ $\left.59.562^{\circ} \mathrm{E}\right), 6-8 . V I I .2010, \mathrm{~K}$. Tomkovich (2 $\sigma^{\top} \sigma^{\top}, 2$ 우, ZMUM); Krasnoselkup $\left(65.7061^{\circ} \mathrm{N} 82.4632^{\circ} \mathrm{E}\right), 16$. VIII.1992, D. Kasparyan ( $3 \mathrm{O}^{7} \mathrm{O}^{7}$, ZISP); the It'yakh River $\left(61.85^{\circ} \mathrm{N} 69.06^{\circ} \mathrm{E}, 61.73^{\circ} \mathrm{N} 71\right.$ $\left.03^{\circ} \mathrm{E}\right), 21$ and 22.VII.2010, K. Tomkovich (2 $\sigma^{\top} \sigma^{\top}$, ZMUM); Sob' env. $\left(67.07^{\circ} \mathrm{N} 65.46^{\circ} \mathrm{E}\right), 26-31 . \mathrm{VII} .2011, \mathrm{~K}$. Tomkovich $\left(1 \sigma^{7}\right.$, ZMUM); the Voykar River Basin (ca. $65.7206^{\circ} \mathrm{N} 64.3244^{\circ} \mathrm{E}$ ), 20.VIII 1925, Fridolin (3 $\sigma^{\top} \sigma^{\top}$, ZISP); the Sob' River Basin (ca. $66.9376^{\circ} \mathrm{N}$ $\left.65.6706^{\circ} \mathrm{E}\right), 4-31$.VII.1925, 4.IX.1925, Fridolin ( 7 O $^{7} \sigma^{7}, 3$ 90, ZISP); the Sob' River Basin (ca. $66.9376^{\circ} \mathrm{N} 65.6706^{\circ} \mathrm{E}$ ), the mouth of the Bol'shaya Pay-Pudyna River, 28.VII.1961, Gorodkov (7 $\sigma^{\top} \sigma^{\top}, 1$, ZISP); Neroyka (ca. $64.57^{\circ} \mathrm{N} 59.67^{\circ} \mathrm{E}$ ), 30.VI.-6.VII.1990, Malozemov (2 $\sigma^{7} \sigma^{7}$, ZISP); Varcha-ty Lake $\left(66.1078^{\circ} \mathrm{N} 63.9871^{\circ} \mathrm{E}\right), 31 . \mathrm{VIII}$. 1 and 4.IX.1925, Fridolin (1 $\sigma^{\top}, 2$ 20 , ZISP); Labytnangi $\left(66.6579^{\circ} \mathrm{N}\right.$
66.3919² E), 28.VII.1973, R. Kamenskaya (1 $\sigma^{7}$, ZMUM); Yakutia: Vilyuysk env. (63.7503 $\left.\mathrm{N} 121.637^{\circ} \mathrm{E}\right), 24$. VIII.1988, Gorodkov (1 + , ZISP); Tiksi $\left(71.6351^{\circ} \mathrm{N} 128.8579^{\circ} \mathrm{E}\right), 9 . \mathrm{VII} .1957$, Gorodkov (1 ऽ, ZISP).

DESCRIPTION. Male (Fig. 1), female. Small or medium-sized flies (3.7-6.2 mm long).

Head. Frontal vitta reddish-yellow, matt; fronto-orbital plate black in upper half and reddish-yellow in lower half, greyish dusted. Ocellar triangle black, with grey pollen. Face and parafacial black to pale yellow in male, completely pale yellow in female. Gena and postgena pale yellow. Postcranium black, greyish dusted, covered with black setae in upper third and white hairs in lower part. 3 orbitals, 2-3 frontals, 1 ocellar, 1 small postocellar, 1 inner vertical, 1 outer vertical; usually 2 pairs of vibrissae present. Antenna black. Postpedicel rounded apically, approximately 2 times as long as wide. Arista black, basally thickened, bare. Clypeus and proboscis black. Palpus broadened towards apex, yellow.

Thorax and scutellum black, greyish dusted. Acrostical hairs in two rows, dorsocentrals (3-4)+3, intraalars $1+(1-2)$, supra-alars $1+2$, postpronotals 2 , notopleurals 2 , postalars 2 . Proepisternum with hairs in central part, with 1-2 long pale setae near lower margin. Proepimeron with one pale seta and usually with several small hairs. Anepisternum covered with pale hairs in posterior half, with 1-3 black setae along posterior margin. Katepisternum covered with pale hairs in posterior half and 1 strong seta in upper posterior corner. Anepimeron bare. Scutellum black, greyish dusted, with a pair of strong basal scutellar and a pair of strong apical scutellar setae.

Legs yellow, only mid and hind coxae black. Fore femur with a row of posterodorsal setae, with numerous hairs anteriorly and ventrally (Fig. 4). Fore tibia with short black ventral spinules in two rows on whole length, with 1 dorsal seta near middle, with 1 preapical dorsal seta and usually with 1 apical posterior seta (Fig. 4). Mid femur with irregular row of anterior setae, with 1 preapical posterior and 1 preapical posterodorsal setae. Mid tibia with 1 anterodorsal and $0-1$ posterodorsal setae near middle, and a ring of apicals. Hind femur with a row of anterodorsal setae. Hind tibia with 2 anterodorsal, 1-2 posterodorsal and 1 preapical dorsal, $0-1$ preapical posteroventral setae, also with 1 apical anterodorsal and 1 apical anteroventral setae.

Wing (Fig. 9) slightly darkened; veins brownish. $\mathrm{R}_{1}$ bare dorsally. Calypters, margins of calypters, and halteres yellowish.

Abdomen black, greyish dusted; female abdominal tergites 6 and 7 sometimes reddish. Male sternite 4 rectangular, about 2 times as wide as long (Fig. 17). Male sternite 5 symmetrical; lobes simple, wide basally, with one row of spinules on inside margin (Fig. 31). Epandrium and surstyli as in Figs 51, 52. Female sternites 5-7 as in Fig. 72; sternite 5 egg-form, narrower than sternite 7.

DISTRIBUTION. Russia (Fig. 84): Arkhangelsk Oblast, Buryatia, Kamchatka Kray, Karelia, Komi, Krasnoyarsk Kray, Leningrad Oblast, Magadan Oblast, Murmansk Oblast, Sakhalin Oblast, Tuva, Tyumen' Oblast, Yakutia. - Europe, North America. 


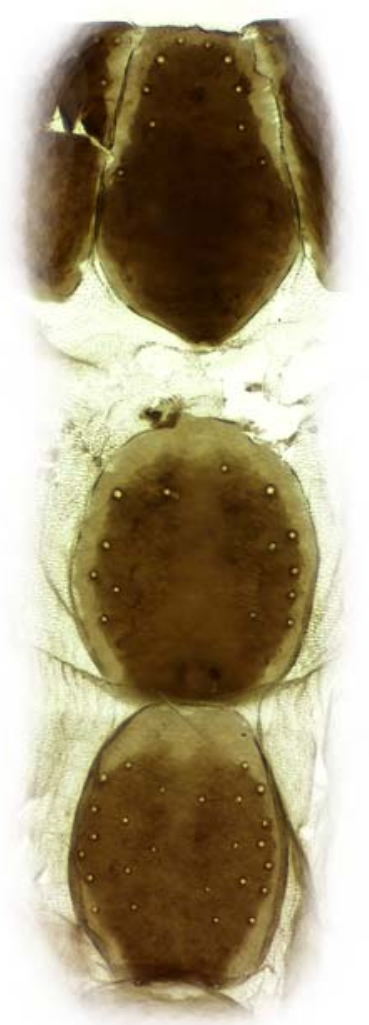

66

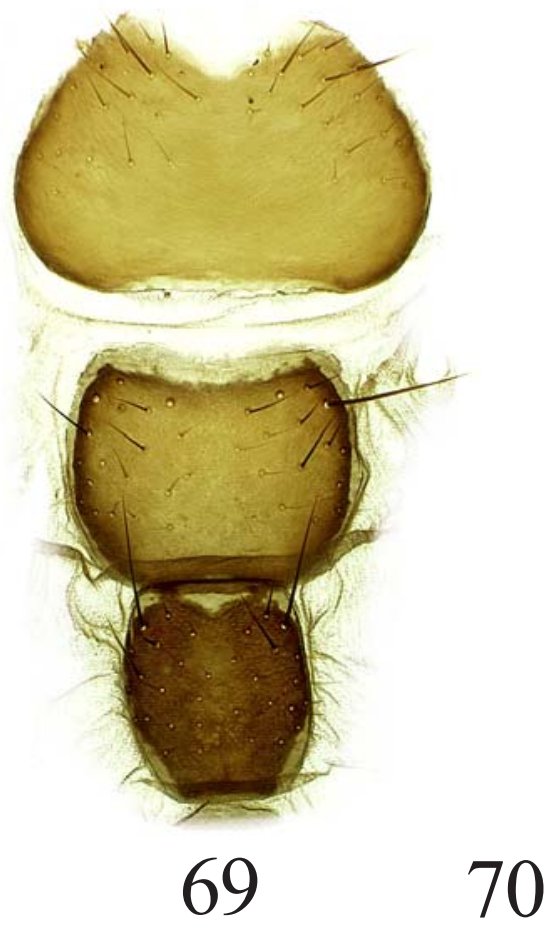

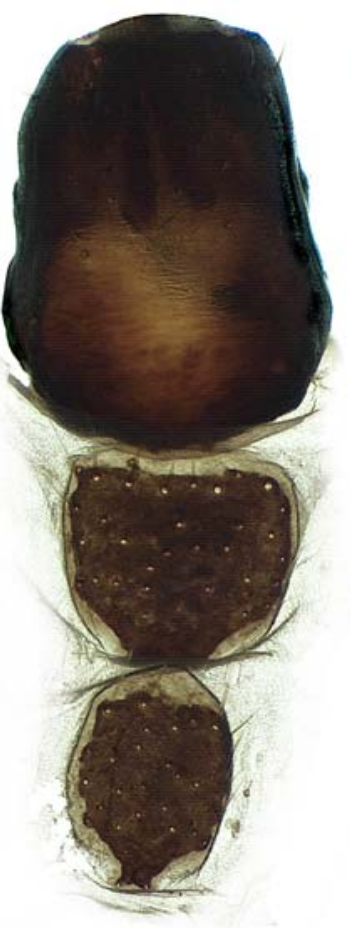

67

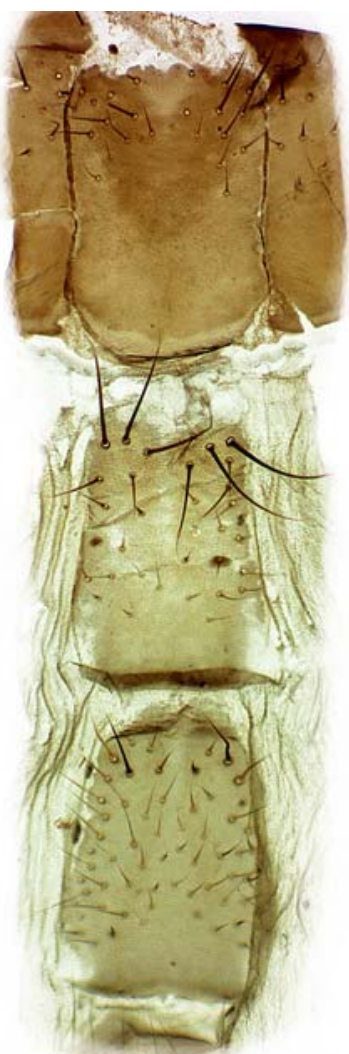

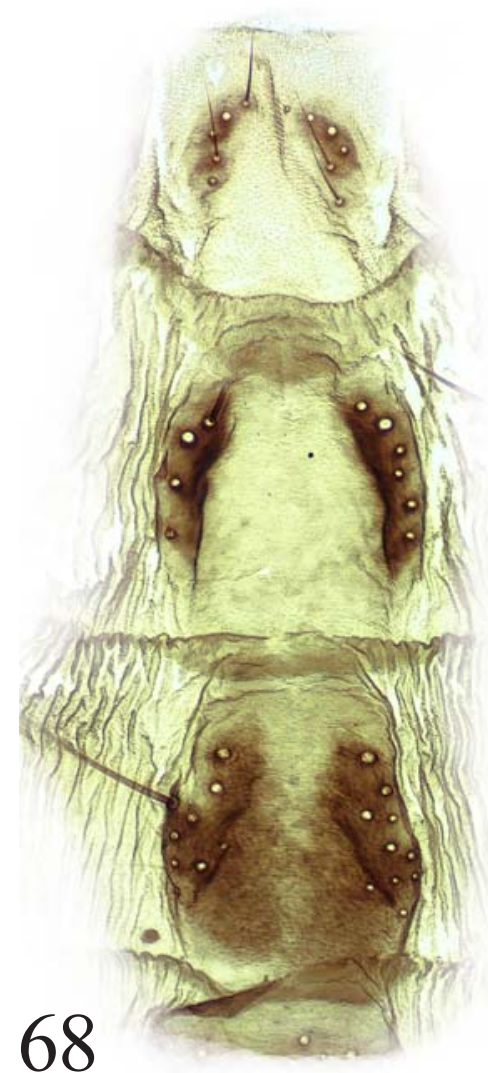

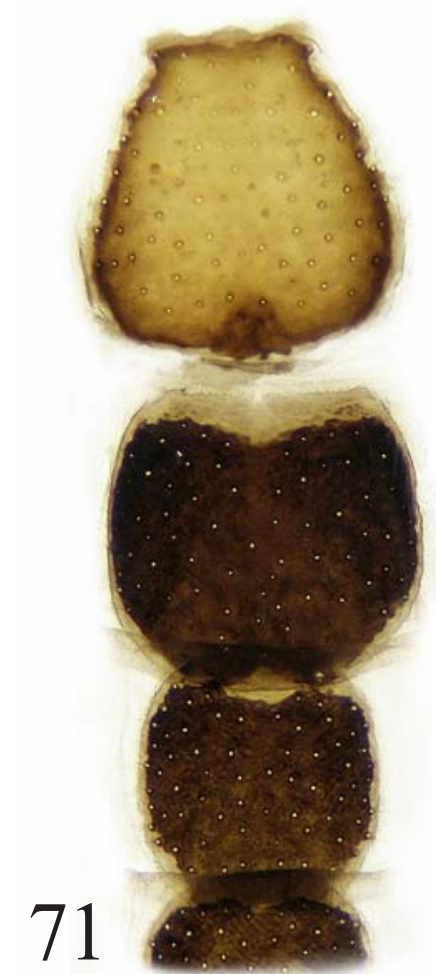

Figs 66-71. Female abdominal sternites 5-7 of Microprosopa: $66-$ M. albipennis (Zetterstedt); 67 - M. crinipes Ringdahl; $68-$ M. frigida (Holmgren); $69-$ M. haemorrhoidalis (Meigen); $70-M$. heteromyzina (Zetterstedt); $71-$ M. lineata (Zetterstedt).

Рис. 66-71. Стерниты 5-7 брюшка самки Microprosopa: $66-$ M. albipennis (Zetterstedt); $67-$ M. crinipes Ringdahl; $68-M$. frigida (Holmgren); $69-$ M. haemorrhoidalis (Meigen); $70-$ M. heteromyzina (Zetterstedt); $71-$ M. lineata (Zetterstedt). 

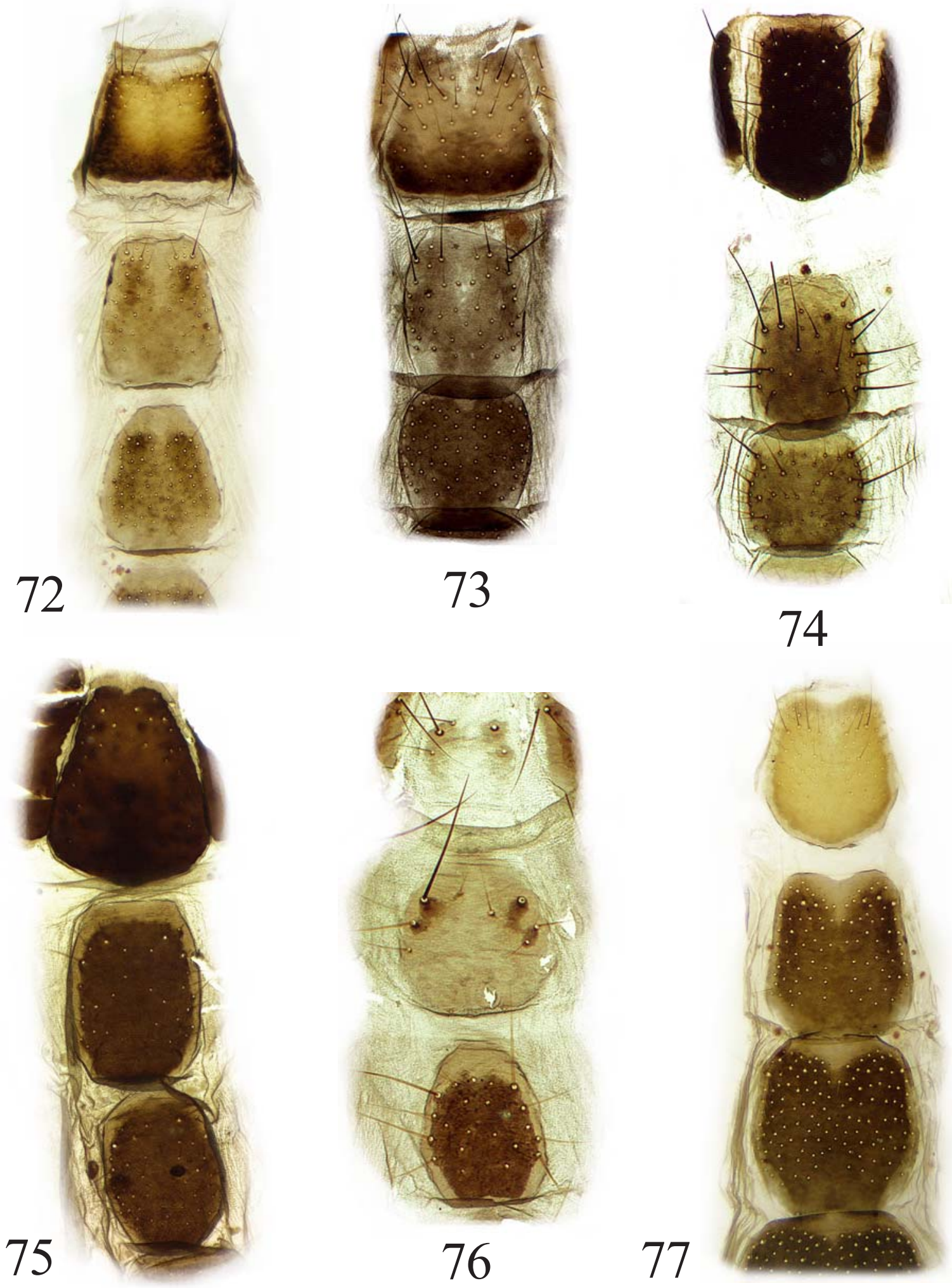

Figs 72-77. Female abdominal sternites 5-7 of Microprosopa: $72-M$. pallidicauda (Zetterstedt); $73-M$. paveli Ozerov et Krivosheina; $74-$ M. portenkoi Stackelberg; $75-$ M. taimyrica sp.n.; $76-$ M. unguiculata (Malloch); 77 - M. zlobini Ozerov.

Рис. 72-77. Стерниты 5-7 брюшка самки Microprosopa: 72 - M. pallidicauda (Zetterstedt); 73 - M. paveli Ozerov et Krivosheina; $74-$ M. portenkoi Stackelberg; $75-$ M. taimyrica sp.n.; $76-$ M. unguiculata (Malloch); 77 — M. zlobini Ozerov. 
Microprosopa (Microprosopa) paveli Ozerov et Krivosheina, 2013

$$
\text { Figs 18, 32, 53, 54, 73, } 78 \text {. }
$$

paveli Ozerov et Krivosheina, 2013: 4 (Microprosopa). Typelocality: Meynipyl'gino (62.5385N 177.0519E) (Russia, Chukotka).

The species was known only from the type-locality.

MATERIAL EXAMINED. Chukotka: Meynypil'gyno env. (62. $\left.6283^{\circ} \mathrm{N} 176.9766^{\circ} \mathrm{E}\right), 14$.VII.2015, P.S. Tomkovich $\left(2 \sigma^{\top} \sigma^{\top}\right.$ ZMUM); Tyumen' Oblast: Neroyka (ca. $\left.64.57^{\circ} \mathrm{N} 59.67^{\circ} \mathrm{E}\right), 29 . \mathrm{VI}$. and 6-9.VII.1990, Malozemov (6 $\sigma^{7} \sigma^{7}, 1$ \%, ZISP).

DESCRIPTION. Male, female. Small or mediumsized flies (4.2-5.0 mm long).

Head. Frontal vitta reddish-yellow, but in upper part blackish, matt; fronto-orbital plate black, greyish dusted. Ocellar triangle black, with grey pollen. Face, including pale yellow part near lower margin and parafacial black in male, completely pale yellow in female. Gena and postgena yellow. Postcranium black, greyish dusted, covered with black setae in upper third and white hairs in lower part. 3 orbitals, 2-3 frontals, 1 ocellar, 1 small postocellar, 1 inner vertical, 1 outer vertical; 1-2 pairs of vibrissae present. Antenna black. Postpedicel rounded apically, approximately 2 times as long as wide. Arista black, basally thickened, bare. Clypeus and proboscis black. Palpus broadened towards apex, yellow.

Thorax and scutellum black, greyish dusted. Acrostical hairs in two rows, dorsocentrals $(3-4)+3$, intraalars $1+2$, supra-alars $1+2$, postpronotals 2 , notopleurals 2, postalars 2. Proepisternum with hairs in central part, with 1-2 long pale setae near lower margin. Proepimeron with one pale seta. Anepisternum covered with pale hairs in posterior half, with 3-4 black setae along posterior margin. Katepisternum covered with pale hairs in posterior half and 1 strong seta in upper posterior corner. Anepimeron bare. Scutellum black, greyish dusted, with a pair of strong basal scutellar and a pair of strong apical scutellar setae.

Legs yellow, fore coxa from yellow to black, mid and hind coxae black, fore femur sometimes darkened posteriorly. Fore femur with a row of posterodorsal setae, with numerous hairs anteriorly and ventrally. Fore tibia with short black ventral spinules in two rows on whole length, with 1 dorsal seta near middle, with 1 preapical dorsal and 1 apical posterior setae. Mid femur with irregular row of anterior setae, with 1 preapical posterior and 1 preapical posterodorsal setae. Mid tibia with 1 anterodorsal seta near middle, and a ring of apicals. Hind femur with a row of anterodorsal setae. Hind tibia with 1-2 anterodorsal, 1 posterodorsal and 1 preapical dorsal setae, also with 1 apical anterodorsal and 1 apical anteroventral setae.

Wing clear or slightly darkened; veins brownish. $\mathrm{R}_{1}$ bare dorsally. Calypters, margins of calypters, and halteres yellowish.

Abdomen black, greyish dusted. Male sternite 4 rectangular, about 2 times as wide as long (Fig. 18). Male sternite 5 symmetrical; lobe inside widening and with two rows of spinules (Fig. 32). Epandrium and surstyli as in Figs 53, 54. Female sternites 5-7 as in Fig. 73; sternite 5 rectangular, not wider than sternite 6 and sternite 7; sternite 7 as long as wide.

DISTRIBUTION. Russia (Fig. 78): Chukotka, Tyumen' Oblast.

Microprosopa (Allomyella) portenkoi Stackelberg, 1952

Figs 19, 34-36, 55, 56, 74, 78.

portenkoi Stackelberg, 1952: 406 (Microprosopa). Type-locality: Wrangel I., Aternon-Rogers (70.9832N 178.4166E) (Russia).

For Russia this species was known only from the type-locality.

MATERIAL EXAMINED. Chukotka: $55 \mathrm{~km} \mathrm{~N}$ of Egvekinot (66.7956 $\left.{ }^{\circ} \mathrm{N} 181.00052^{\circ} \mathrm{E}\right), 29$. VII.1963, Gorodkov (2 $\sigma^{7} \sigma^{7}$, ZISP); Krasnoyarsk Kray: Noril'skie Mt. $\left(69.2332^{\circ} \mathrm{N} 88.2545^{\circ} \mathrm{E}\right)$, the Savel'evka River, 28.VII.1967, Grunin (1 $\sigma^{7}$, ZISP).

DESCRIPTION. Male, female. Small-sized flies (3.2-4.1 mm long).

Head. Frontal vitta black, only in lower third or quarter yellow, matt; fronto-orbital plate and ocellar triangle black, greyish dusted. Face blackish, yellowish along margin of mouth only. Parafacial blackish. Gena yellow, postgena black, greyish dusted. Postcranium black, greyish dusted, covered with black setae in upper third and pale hairs in lower part. 3 orbitals, 2-4 frontals, 1 ocellar, 1 postocellar, 1 inner vertical, 1 outer vertical; $1-2$ pairs of vibrissae present. Antenna black. Postpedicel with slightly acute upper apical corner, 1.5-2.0 times as long as wide. Arista black, basally thickened, bare. Clypeus and proboscis black. Palpus broadened towards apex, yellow.

Thorax and scutellum black, greyish dusted. Acrostical hairs in two rows, dorsocentrals $(3-4)+3$, intraalars $1+2$, supra-alars $1+2$, postpronotals 2 , notopleurals 2, postalars 2. Proepisternum with hairs in central part, with 1-2 pale setulae near lower margin. Proepimeron with one pale seta. Anepisternum covered with pale hairs in posterior half, with 1-3 black setae along posterior margin. Katepisternum covered with pale hairs in posterior half and 1 strong seta in upper posterior corner. Anepimeron usually with a few hairs, but sometimes bare. Scutellum black, greyish dusted, with a pair of basal scutellar and a pair of apical scutellar setae.

Coxae, trochanters and femora of all legs black; tibiae and tarsi of all legs from yellow to blackish. Fore femur with a row of posterodorsal setae, with pale hairs posteroventrally. Fore tibia with short black spinules ventrally in two rows on whole length, with 1 dorsal and 1 posterior setae near middle, with 1 preapical dorsal seta and 1 apical posterior seta. Mid femur with irregular row of anterior setae in apical half or $2 / 3$, with 1 preapical posterior and 1 preapical posterodorsal setae, also with pale hairs ventrally. Mid tibia with 1 anterodorsal, $0-1$ posterodorsal, and 1 posterior setae near middle, also with a ring of apicals. Hind femur with a row of black anterodorsal and a row of usually pale anteroventral setae. Hind tibia with 2 anterodor- 
sal, 1-2 posterodorsal and 1 preapical dorsal, 0-1 preapical posterodorsal setae, also with 1 apical anteroventral seta.

Wing slightly darkened, sometimes darkened along $\mathrm{r}-\mathrm{m}$ and dm-cu crossveins; veins blackish. $\mathrm{R}_{1}$ bare dorsally. Calypters, margins of calypters, and halteres whitish or yellowish.

Abdomen black, tergites 3-6 greyish dusted medially and shining along lateral margins; female tergite 6 shining along basal margin. Male sternite 4 rectangular, about 2 times as wide as long (Fig. 19). Male sternite 5 asymmetrical, lobes bifurcate apically (Figs 34-36). Epandrium and surstyli as in Figs 55, 56. Female sternites $5-7$ as in Fig. 74; sternites 6 and 7 sclerotized completely.

DISTRIBUTION. Russia (Fig. 78): Chukotka, Krasnoyarsk Kray. — North America.

\section{Microprosopa (Allomyella) taimyrica sp.n.} Figs 6, 10, 20, 37, 57, 58, 64, 75, 78.

MATERIAL EXAMINED. Holotype $\sigma^{7}$, Krasnoyarsk Kray, Lake Engel'gardt $\left(75.101^{\circ} \mathrm{N} 100.2344^{\circ} \mathrm{E}\right), 2 . \mathrm{VIII} .1967$, Gorodkov (ZISP). Paratype + , same labels (ZMUM).

DESCRIPTION. Male, female. Small-sized flies (4.3-4.4 mm long).

Head. Frontal vitta black, only in lower third (male) or quarter (female) yellow, greyish dusted; fronto-orbital plate and ocellar triangle black, greyish dusted. Face and parafacial yellow. Gena yellow, postgena black. Postcranium black, greyish dusted, covered with black setae in upper third and pale hairs in lower part. 3 orbitals, 2-3 frontals, 1 ocellar, 1 small postocellar, 1 inner vertical, 1 outer vertical; 1 or 2 pairs of vibrissae present. Antenna yellow, postpedicel in female darkened apically and along anterior margin. Postpedicel rounded apically, approximately 2 times as long as wide. Arista yellowish (male) or brown (female), basally thickened, bare. Clypeus and proboscis black. Palpus broadened towards apex, yellow.

Thorax and scutellum black, greyish dusted. Acrostical hairs in two irregular rows, dorsocentrals (3-4)+3, intra-alars $1+2$, supra-alars $1+2$, postpronotals 2 , notopleurals 2, postalars 2. Proepisternum with hairs in central part, with 1-2 long pale setae near lower margin. Proepimeron with 1 long pale seta. Anepisternum covered with pale hairs in posterior half, with 1-3 black setae along posterior margin. Katepisternum covered with pale hairs in posterior half and 1 strong seta in upper posterior corner. Anepimeron bare. Scutellum black, greyish dusted, with a pair of basal scutellar and a pair of apical scutellar setae.

Coxae and femora of all legs black, tibiae and tarsi yellow. Fore femur with rows of posterodorsal and posteroventral setae, with pale hairs posteriorly and posteroventrally. Fore tibia with short black spinules ventrally in two rows on whole length, with 1 dorsal and 1 posterior setae near middle, with 1 preapical dorsal and 1 apical anterior setae. Mid femur with irregular row of anterior setae in apical half and a row of posteroventral setae, with 1 preapical posterior and 1 preapical posterodorsal setae. Mid tibia with 1 anterodorsal and 1 posterior setae near middle, and a ring of apicals. Hind femur with rows of anterodorsal, anteroventral and posteroventral setae. Hind tibia with 2 anterodorsal, 1 preapical dorsal, and 1 apical anteroventral setae; additionally with long setae on anteroventral surface in male (Fig. 6) and several short anteroventral setae at middle in female.

Wing slightly yellowish, darkened along longitudinal veins (Fig. 10). Crossveins r-m and dm-cu also distinctly darkened. Veins brown. $\mathrm{R}_{1}$ bare dorsally. Calypters, margins of calypters, and halteres yellowish.

Abdomen black greyish dusted, tergites 3-5 shining along lateral margins. Male sternite 4 rectangular, about 2 times as wide as long (Fig. 20). Male sternite 5 symmetrical, lobes bifurcate apically (Fig. 37). Epandrium and surstyli as in Figs 57, 58. Female sternites 5-7 as in Fig. 75; sternite 7 ovoid, longer than wide.

COMPARISON. The new species resembles $M i$ croprosopa crinipes Ringdahl, 1928. The differences between these species are given in the identification key below.

DISTRIBUTION. Russia (Fig. 78): Krasnoyarsk Kray (Taimyr). 1919)

Microprosopa (Allomyella) unguiculata (Malloch,

Figs 21, 38, 59, 60, 76, 85 .

unguiculata Malloch, 1919: 80 (Allomyia). Type-locality: Chantry Island, Bernard Harbour, Dolphin and union strait, Northwest Territories (Canada).

borealis Curran, 1927: 260 (Allomyella). Type-locality: Cape Prince of Wales, Alaska (USA) - syn.n.

The species was recorded by Ozerov \& Barkalov [2014: 561 (as Allomyella)] from Krasnoyarsk Kray (Taymyr).

MATERIAL EXAMINED. Yakutia: airport Saskylakh (71. $\left.9345^{\circ} \mathrm{N} 114.083^{\circ} \mathrm{E}\right), 24$.VII.1988, Gorodkov (1 $\sigma^{7}$, ZISP);

ADDITIONAL MATERIAL EXAMINED. Holotype of Allomyella borealis Curran, + , with labells: 1) "C. Prince Wales Alaska 27 VI 1926 D Jenness", 2) "Allomyella borealis Curran $q$ TYPE No.2619" (CNC). Abdomen was dissected for study abdominal sternites and found to be conspecific with Microprosopa unguiculata (Malloch, 1919).

DESCRIPTION. Male, female. Small-sized flies (3.3-3.6 mm long).

Head. Frontal vitta black, only in lower quarter yellow, greyish dusted; fronto-orbital plate and ocellar triangle black, greyish dusted. Face blackish in upper half and yellowish in lower half. Parafacial blackish. Gena black, greyish dusted. Postcranium black, greyish dusted, covered with black setae in upper third and pale hairs in lower part. 3 orbitals, 2-3 frontals, 1 ocellar, 1 small postocellar, 1 inner vertical, 1 outer vertical; 1 pair of vibrissae present. Antenna black. Postpedicel with slightly acute upper apical corner, approximately 2 times as long as wide. Arista black, basally thickened, bare. Clypeus and proboscis black. Palpus broadened towards apex, yellow.

Thorax and scutellum black, greyish dusted. Acrostical hairs in two rows, dorsocentrals $4+3$, intra-alars 


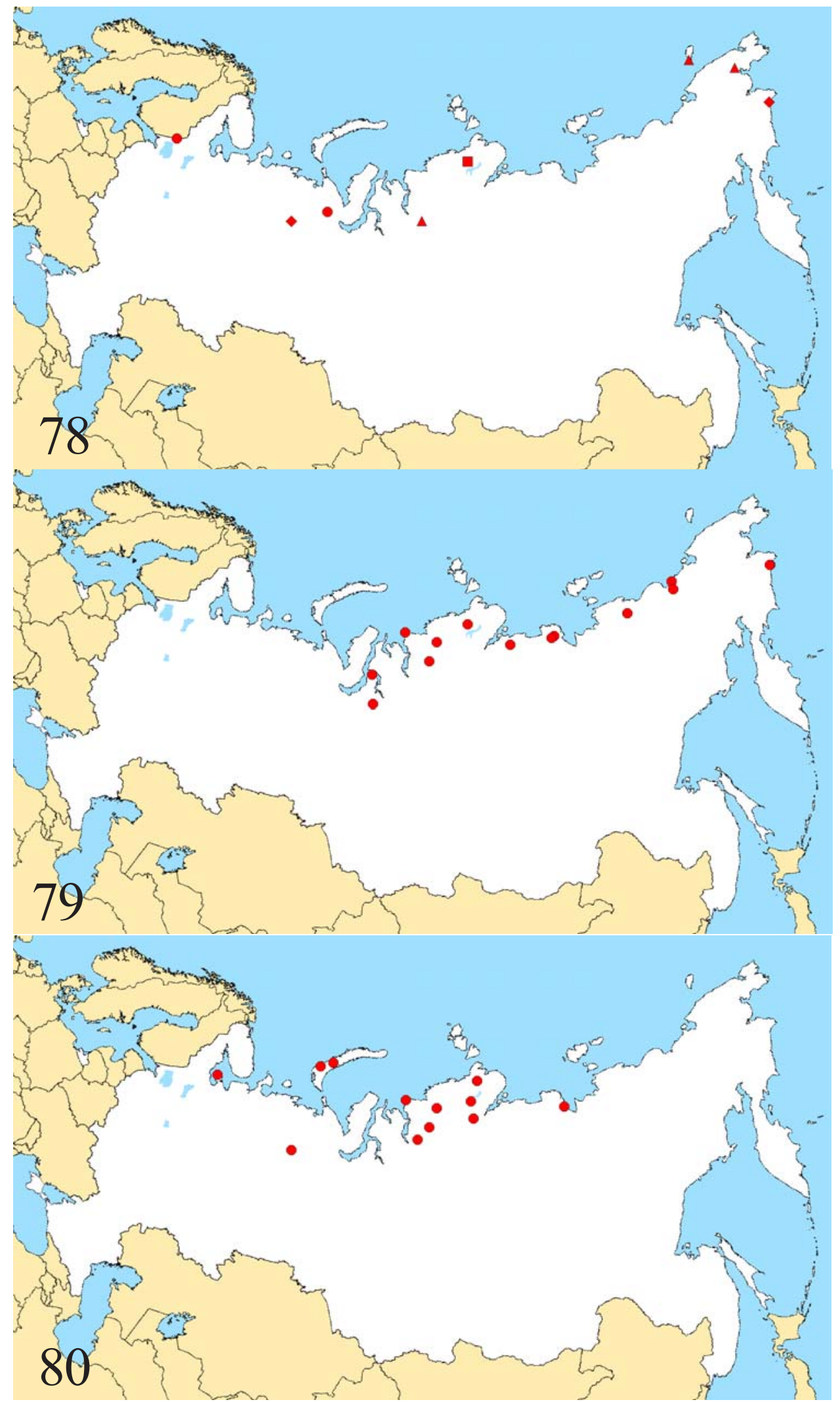

Figs 78-80. Distribution maps of Microprosopa: $78-$ M. albipennis (Zetterstedt) (•), M. paveli Ozerov et Krivosheina $(\bullet), M$.

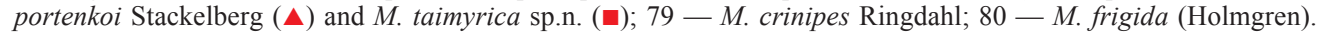

Рис. 78-80. Распространение видов рода Microprosopa: $78-$ M. albipennis (Zetterstedt) (•), M. paveli Ozerov et Krivosheina

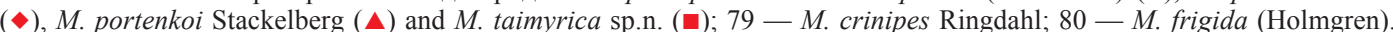




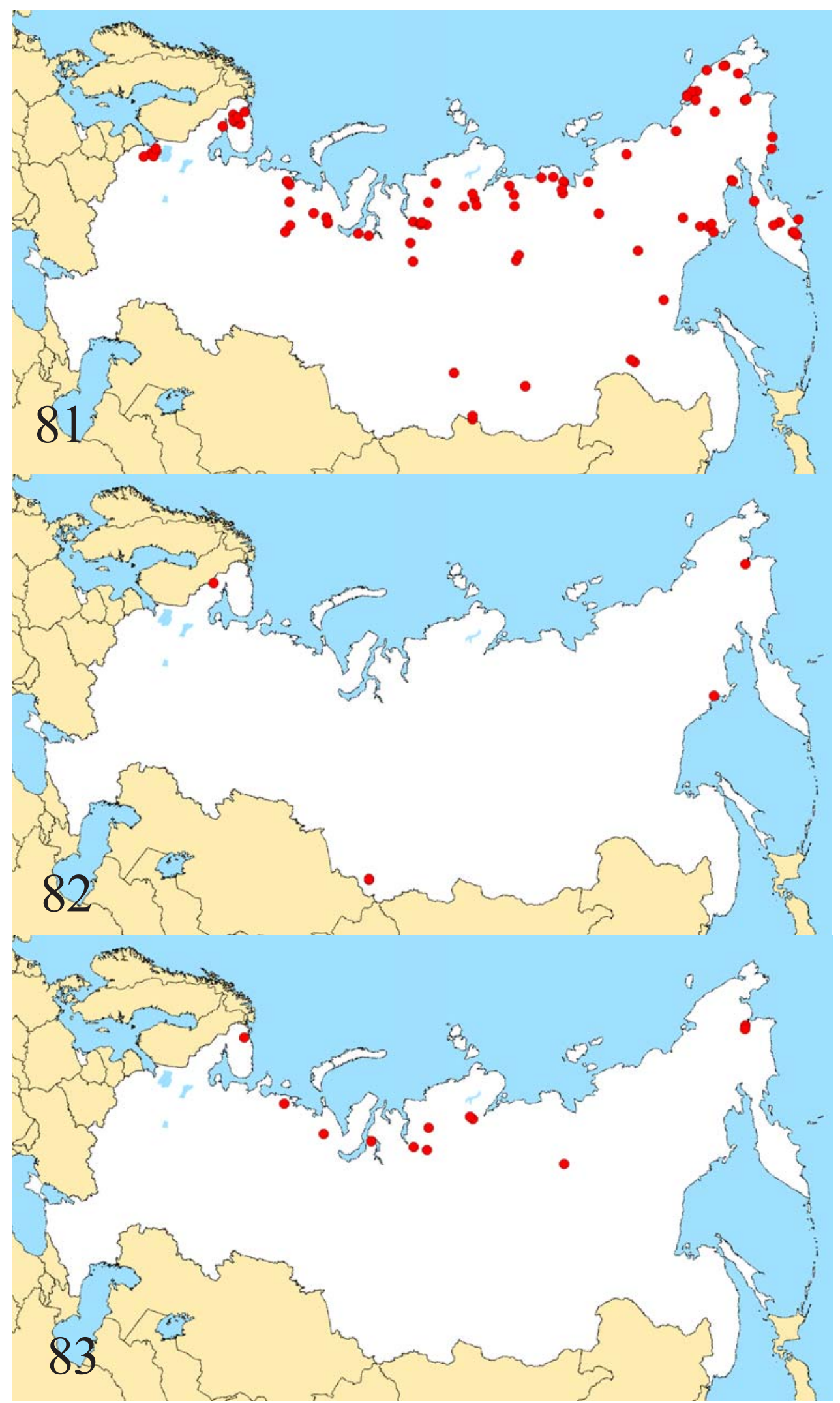

Figs 81-83. Distribution maps of Microprosopa: $81-$ M. haemorrhoidalis (Meigen); $82-$ M. heteromyzina (Zetterstedt); $83-M$. lineata (Zetterstedt).

Рис. 81-83. Распространение видов рода Microprosopa: $81-$ M. haemorrhoidalis (Meigen); $82-$ M. heteromyzina (Zetterstedt); $83-$ M. lineata (Zetterstedt). 


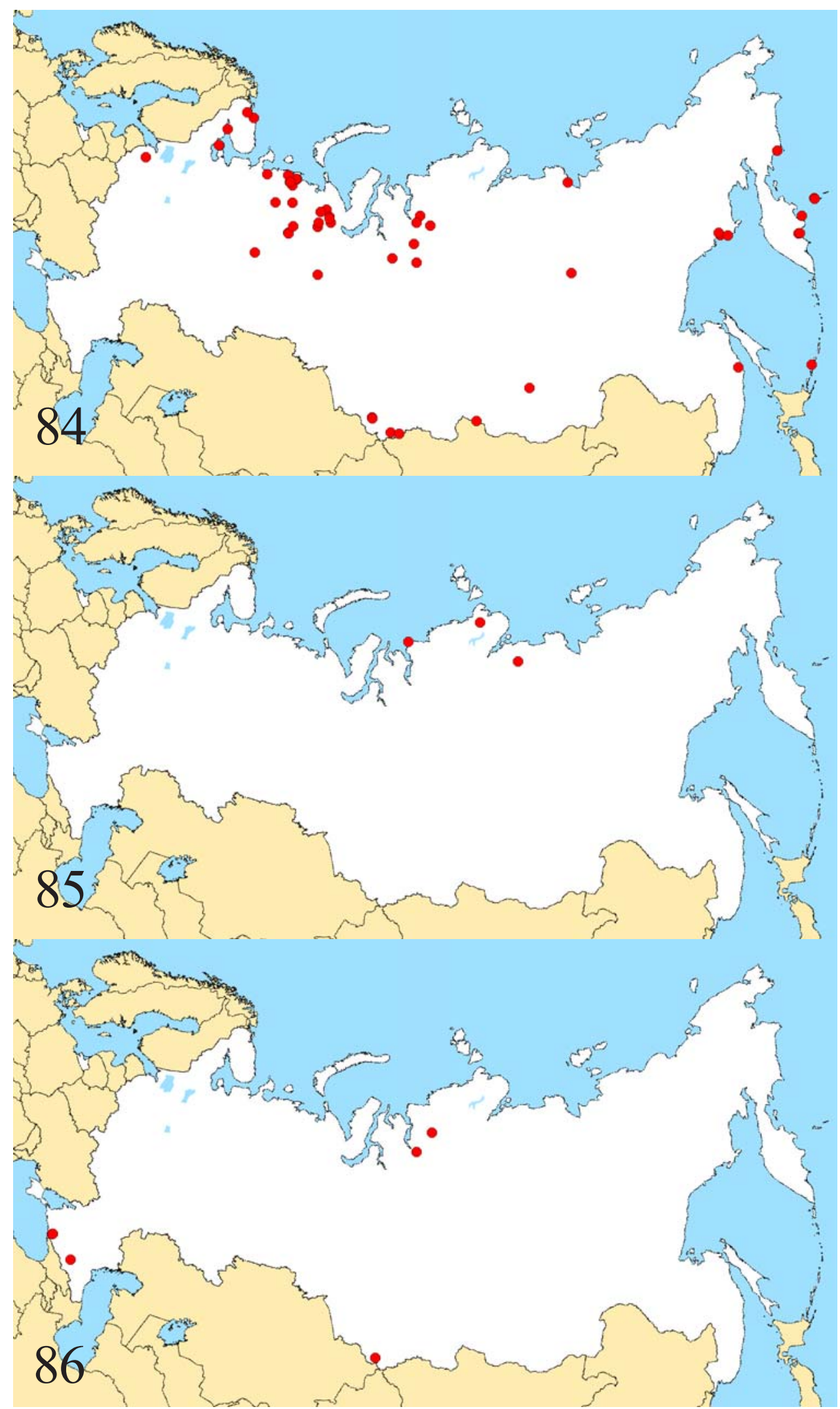

Figs 84-86. Distribution maps of Microprosopa: $84-$ M. pallidicauda (Zetterstedt); $85-$ M. unguiculata (Malloch); $86-M$. zlobini Ozerov.

Рис. 84-86. Распространение видов рода Microprosopa: 84 - M. pallidicauda (Zetterstedt); 85 — M. unguiculata (Malloch); 86 - M. zlobini Ozerov. 
$1+2$, supra-alars $1+2$, postpronotals 2 , notopleurals 2 , postalars 2. Proepisternum with hairs in central part, with long pale seta near lower margin. Proepimeron with one long pale hair and a few small hairs. Anepisternum covered with pale hairs in posterior half, with 2-4 black setae along posterior margin. Katepisternum covered with pale hairs in posterior half and 1 strong seta in upper posterior corner. Anepimeron usually with a few hairs, but sometimes bare. Scutellum black, greyish dusted, with a pair of basal scutellar and a pair of apical scutellar setae.

Legs black, but fore tibia, usually mid tibia and all tarsi yellowish. Fore femur with a row of posterodorsal setae, with long pale hairs posteroventrally. Fore tibia with short black spinules ventrally in two rows on whole length, with 1 dorsal and 1 posterior setae near middle, 1 preapical dorsal seta, and also with apical anterodorsal and apical posterior setae. Mid femur with irregular row of anterior setae in apical half, with 1 preapical posterior and 1 preapical posterodorsal setae. Mid tibia with 1 anterodorsal and 1 posterodorsal setae near middle, 1-2 posterior setae in apical third, and a ring of apicals. Hind femur with a row of black anterodorsal and a row of pale anteroventral setae. Hind tibia with 2 anterodorsal, 1-2 posterodorsal and 1 preapical dorsal, 1 preapical posteroventral setae, also with 1 apical anterodorsal and 1 apical anteroventral setae.

Wing slightly darkened; veins blackish. $\mathrm{R}_{1}$ bare dorsally. Calypters, margins of calypters, and halteres whitish or yellowish.

Abdomen black, greyish dusted; female tergites 7 and 8 shining. Male sternite 4 rectangular, about 2.5 times as wide as long (Fig. 21). Male sternite 5 symmetrical, lobes bifurcate apically (Fig. 38). Epandrium and surstyli as in Figs 59, 60. Female tergite 8 as whole semicircular sclerite, not devided medially on two sclerites. Female sternite 6 circular; sternite 7 small, circular, poorly sclerotized in median part (Figs 76).

DISTRIBUTION. Russia (Fig. 85): Krasnoyarsk Kray, Yakutia. — North America.

Microprosopa (Microprosopa) zlobini Ozerov, 2009 Figs 22, 33, 61, 62, 77, 86.

zlobini Ozerov, 2009: 421 (Microprosopa). Type-locality: North Ossetia, environs of Buron (Tsey gorge [42.793055N, 43.922161E, alt. $1764 \mathrm{~m}$ ) (Russia).

For Russia this species was known only from the type-locality [Ozerov, 2009: 421, 422].

MATERIAL EXAMINED. Altay: Kuzuyak pass $\left(50.115556^{\circ} \mathrm{N}\right.$ $\left.86.410833^{\circ} \mathrm{E}\right), 26$. VI.2007, O. Kosterin (1 $\sigma^{7}$, ZMUM); Krasnodar Kray: Lagonaki $\left(44.01^{\circ} \mathrm{N} 39.99^{\circ} \mathrm{E}\right), 1500-1900 \mathrm{~m}, 5-9 . \mathrm{VI}$ 2015, N. Vikhrev (1 ${ }^{\top}$, ZMUM); Lagonaki $\left(44.093^{\circ} \mathrm{N} 40.019^{\circ} \mathrm{E}\right)$, 1725 m, 26-28.VI.2009, K. Tomkovich (1 오, ZMUM); Krasnoyarsk Kray: Agapa (ca. $\left.71.412^{\circ} \mathrm{N} 89.2689^{\circ} \mathrm{E}\right)$, the Pyasina River 14.VII.1967, Gorodkov (2 $\sigma^{7} \sigma^{7}, 1$ \%, ZISP); Dudinka $\left(69.4042^{\circ} \mathrm{N}\right.$ $\left.86.1822^{\circ} \mathrm{E}\right), 3$. VII.1967, Gorodkov (1 $0^{\top}$, ZISP); North Ossetia Alania: Buron env. $\left(43.7932^{\circ} \mathrm{N} 43.9225^{\circ} \mathrm{E}\right), 6 . \mathrm{VI} .1989$ and 3-5. VII.1990, A.L. Ozerov (12 $0^{7} \sigma^{7}, 3$ 우, ZMUM).

DESCRIPTION. Male, female. Small or mediumsized flies (3.8-5.9 mm long).
Head. Frontal vitta reddish-yellow, matt; frontoorbital plate greyish dusted. Ocellar triangle black, with grey pollen. Face blackish in upper half and white in lower half in male, completely white in female. Parafacial blackish in male and white in female. Gena pale yellow, matt. Postcranium black, greyish dusted, covered with black setae in upper third and white hairs in lower part. 3 orbitals, 2-3 frontals, 1 ocellar, 1 postocellar, 1 inner vertical, 1 outer vertical; $1-2$ pairs of vibrissae present. Scape and pedicel dark brown to black. Postpedicel black, rounded apically, approximately 2 times as long as wide. Arista black, basally thickened and very short haired, apically bare. Clypeus and proboscis black. Palpus broadened towards apex, yellow.

Thorax and scutellum black, greyish dusted. Acrostical hairs in two rows, dorsocentrals $(4-5)+3$, intra-alars $1+(1-2)$, supra-alars $1+2$, postpronotals 2 , notopleurals 2 , postalars 2. Proepisternum with hairs in central part, with long pale seta near lower margin. Proepimeron with one long pale hair and several small hairs. Anepisternum covered with pale hairs in posterior half, with 2-3 black setae along posterior margin. Katepisternum covered with pale hairs in posterior half and 1 strong seta in upper posterior corner. Anepimeron bare. Scutellum black, greyish dusted, with a pair of strong basal scutellar and a pair of strong apical scutellar setae.

Legs yellow. Fore femur with a row of posterodorsal setae, with numerous small hairs anteriorly and ventrally. Fore tibia with short black spinules ventrally in two rows on whole length, with 1 dorsal seta near middle, with 1 preapical dorsal seta and apical anterodorsal and apical posterior setae. Mid femur with irregular row of anterior setae, with 1 preapical posterior and 1 preapical posterodorsal setae. Mid tibia with 1 anterodorsal and $0-1$ posterior setae near middle, and a ring of apicals. Hind femur with a row of anterodorsal setae. Hind tibia with 2-3 anterodorsal, 1-2 posterodorsal and 1 preapical dorsal setae, also with 1 apical anterodorsal and 1 apical anteroventral setae.

Wing slightly darkened; veins brownish. $\mathrm{R}_{1}$ bare dorsally. Calypters, margins of calypters, and halteres yellowish.

Abdomen black, greyish dusted. Male sternite 4 rectangular, about 1.5 times as wide as long (Fig. 22). Male sternite 5 symmetrical, lobes with double row of spinules (Fig. 33). Epandrium and surstyli as in Figs 61, 62. Female sternites 5-7 as in Fig. 77; sternite 5 oval, width approximately equal to width of sternite 7 .

DISTRIBUTION. Russia (Fig. 86): Altay, Krasnodar Kray, Krasnoyarsk Kray, North Ossetia-Alania. — Europe (Switzerland) [Ozerov, 2009: 422].

\section{Key to the SPECIES OF MicRoprosopa OF RUSSIA}

1. Scutum with two shining narrow stripes medially and fore shining spots (Fig. 3). Male sternites 4 and 5 as in Figs 16, 30; epandrium, cerci and surstyli as in Figs 49, 50. Female sternites 5-7 as in Fig. 71

M. lineata (Zetterstedt) 
- Scutum without shining narrow stripes medially, as a rule grey dusted completely ................................................ 2

2. Femora of all legs yellow. Lobes of male sternite 5 symmetrical, simple (Fig. 28-33) ........................................ 3

- Femora of all legs black. Lobes of male sternite 5 symmetrical or asymmetrical, bifurcate apically or with three horns (Fig. 23-27, 34-38)

3. Postcranium yellow in lower quarter. Abdomen in female usually yellow completely. Male sternites 4 and 5 as in Figs 15, 29; epandrium, cerci and surstyli as in Figs 47, 48. Female sternites $5-7$ as in Fig. 70

M. heteromyzina (Zetterstedt)

- Postcranium entirely black. Female abdomen dark brown or black, tergite 6 only sometimes yellow

4. Male sternites 4 and 5 as in Figs 14, 28. Epandriur and surstyli as in Figs 45, 46. Female abdominal tergite 6 yellow; sternite 7 wide, broader than long (Fig. 69) ..

M. haemorrhoidalis (Meigen)

- Male sternites 4 and 5 as in Figs 17, 18, 22, 31-33. Female abdominal tergite 6 yellow (some specimens of M. pallidicauda) or black; sternite 7 narrow, longer than wide (Fig. 72, 73, 77)

5. Width of male sternite 4 about 2 times as wide as long (Fig. 17); lobes of sternite 5 with one row of spinules (Fig. 31). Epandrium, cerci and surstyli as in Figs 51, 52. Female sternites 5-7 as in Fig. 72; sternite 5 egg-form, narrower than sternite 7 .................... M. pallidicauda (Zetterstedt)

- Width of male sternite 4 about 1.5 times as wide as long (Fig. 22), lobes of sternite 5 wide, with two rows of spinules (Fig. 33). Epandrium, cerci and surstyli as in Figs 61,62 . Female sternites $5-7$ as in Fig. 77; sternite 5 oval, width approximately equal to width of sternite 7 ...

M. zlobini Ozerov

- Width of male sternite 4 about 2 times as wide as long (Fig. 18), lobes of sternite 5 narrow, with two rows of spinules (Fig. 32). Epandrium, cerci and surstyli as in Figs 53, 54. Female sternites $5-7$ as in Fig. 73; sternite 5 egg-form, wider than sternite 7 .......... M. paveli Ozerov

6. Male hind tibia with long hairs anteroventrally (Figs 5, 6). Female hind tibia with several short anteroventral setae at middle

- Male hind tibia without long hairs anteroventrally. Female hind tibia with $0-1$ anteroventral seta only ......... 8

7. Antennae yellow. Anepisternum bare. Wing slightly yellowish, darkened along longitudinal veins (Fig. 10), crossveins $\mathrm{r}-\mathrm{m}$ and $\mathrm{dm}$-cu also distinctly darkened. Male sternites 4 and 5 as in Figs 20, 37. Epandrium, cerci and surstyli as in Figs 57, 58. Female sternite 7 as separate sternite (Fig. 64, 75) .......... M. taimyrica Ozerov, sp.n.

- Antennae black. Anepisternum with a few fine hairs. Wing clear or slightly darkened, crossveins $\mathrm{r}-\mathrm{m}$ and $\mathrm{dm}$ cu not darkened (Fig. 7). Male sternites 4 and 5 as in Figs 12, 24. Epandrium, cerci and surstyli as in Figs 41, 42. Lateral margins of female sternite 7 fused with lateral margins of sternite 7 forming syntergosternite (Fig. 63, 67) ....................................... M. crinipes Ringdahl

8. Mid tibia without seta posteriorly. Male sternites 4 and 5 as in Figs 11,23. Epandrium, cerci and surstyli as in Figs 39, 40. Female sternites 5-7 as in Fig. 66

$$
\text { M. albipennis (Zetterstedt) }
$$

- Mid tibia with posterior seta

9. Lobes of male sternite 5 symmetrical (Fig. 38). Female sternite 6 sclerotized completely, sternite 7 medially sclerotized weakly (Fig. 76). Male sternite 4 as in Fig. 21. Epandrium, cerci and surstyli as in Figs 59, 60
- Lobes of male sternite 5 asymmetrical (Figs 25-27, 3436). Both female sclerites 6 and 7 medially sclerotized weakly (Fig. 68) or both these sclerites sclerotized completely (Fig. 74) ..................................................... 10

10. Left lobe of male sternite 5 with three horns (Fig. 26). Female sternites 6 and 7 medially sclerotized weakly (Fig. 68). Male sternite 4 as in Fig. 13. Epandrium, cerci and surstyli as in Figs 43, 44 ..... M. frigida (Holmgren)

- Left lobe of male sternite 5 with two horns (Fig. 35). Female sternites 6 and 7 sclerotized completely (Fig. 74). Male sternite 4 as in Fig. 19. Epandrium, cerci and surstyli as in Figs 55, 56....... M. portenkoi Stackelberg

ACKNOWLEDGEMENTS. The work was conducted within the state project No AAAA-A16-116021660077-3. Research trip to the Zoological Institute (St. Petersburg), morphological studies and illustrations for this work were made on equipment purchased with the support of RSF, research project No.14-50-00029. I am grateful to Dr. Ol'ga Ovchinnikova and Mrs. Galina Suleymanova for the help with the collection during our work in ZISP.

I am very grateful to Mr. Jere Kahanpää (MZH) for loans of female of Microprosopa albipennis (Zetterstedt), Dr. Jim O'Hara (CNC) for loans of holotype of Allomyella borealis Curran, and Dr. Rune Bygebjerg (MZLU) for loans of syntype of Cordylura melanura Zetterstedt and holotype of Scatomyza heteromyzina (Zetterstedt).

\section{References}

Becker T. 1894. Dipterologische Studien. I. Scatomyzidae // Berliner Entomologische Zeitschrift. Bd.39. H.1. S.77-196.

Becker T. 1897. Beitrag zur Dipteren-Fauna von Nowaja-Semlja // Annuaire du Musée Zoologique de l'Académie Impériale des Sciences de St. Pétersbourg. T.2. P.396-404.

Becker T. 1905. Holometopa. Acalyptera. Scatomyzidae // Becker T., Bezzi M., Kertész K., Stein P. (eds.). Katalog der paläarktischen Dipteren. Vol.4. Budapest. P.1-21.

Cumming J.M., Wood D.M. 2009. Adult morphology and terminology // Brown B.V., Borkent A., Cumming J.M., Wood D.M., Woodley N.E., Zumbado M. (eds.). Manual of Central American Diptera. Vol.1. National Research Council Press, Ottawa. P.9-50.

Curran C.H. 1927. Some new Canadian Scatophagidae (Diptera) // The Canadian Entomologist. Vol.59. No.11. P.253-261.

Engelmark R. 1999. Dungflies (Diptera: Scathophagidae) collected by the Swedish-Russian tundra ecology expedition '94, with the description of two new species; Nanna indotatum and Cochliarium sibiricum // Entomologisk Tidskrift. Arg.120. Häft 4. P.157-167.

Gorodkov K.B. 1970. [Family Scathophagidae (Cordyluridae, Scatomyzidae, Scopeumatidae)] // Bei-Bienko G.Y. (ed.). Key to the insects of the European part of the USSR. Vol.5. Diptera, Siphonaptera. Part 2. P. 440-458 [in Russian].

Gorodkov K.B. 1986. Family Scathophagidae // Soós Á, Papp L. (eds.). Catalogue of Palaearctic Diptera. Vol.11. Scathophagidae-Hypodermatidae. Budapest: Akadémiai Kiadó. P.1141 .

Hackman W. 1956. The Scatophagidae (Dipt.) of Eastern Fennoscandia // Societas pro Fauna et Flora Fennica. Fauna fennica II. Helsingforsiae. $67 \mathrm{pp}$.

Hadley A. 2007. CombineZM. Open source image processing software package for creating extended depth of field images. Available from: http://www.hadleyweb.pwp.blueyonder.co.uk/ (accessed 17 January 2011).

Holmgren A.E. 1883. Diptera // Holmgren A.E. \& Aurivillius C. Insecta a viris doctissimis Nordenskiold illum ducem sequentibus in insulis Waigatsch et Novaja Semlia anno 1878 collecta. 
Hymenoptera et Diptera // Entomologisk Tidskrift. Haft.4. S.162-190.

Humala A.E., Polevoi A.V. 2009. On the Insect fauna of south-east Karelia // Trudi Karelskogo nauchnogo centra RAN. Vol.4. P. 53-75.

Malloch J.R. 1919. The Diptera collected by the Canadian Expedition, 1913-1918 (Excluding the Tipulidae and Culicidae) // Anderson R.M. (ed.). Report of the Canadian Arctic Expedition 1913-18. Vol.3. Insects. Part C: Diptera. Ottawa. P.34 90.

Malloch J.R. 1920. XII. Descriptions of Diptera of the families Anthomyidae and Scatophagidae // The Ohio Journal of Science. Vol.20. No.7. P.267-391.

Malloch J.R. 1923. Diptera (except Tipulidae, Rhyphidae and Calliphoridae) // A biological survey of the Pribilof Islands, Alaska. Part II. Insects, arachnids, and chilopods of the Pribilof Islands, Alaska. North American Fauna. No.46. P.170-227. pls. $12-15$.

McAlpine J.F. 1981. Morphology and terminology-adults // McAlpine J.F., Peterson B.V., Shewell G.E, Teskey H.J., Vokeroth J.R., Wood D.M., Coordinators. Manual of Nearctic Diptera. Vol.2. Research Branch. Agriculture Canada. Monograph 27. Ottawa. P.9-63.

Meigen J.W. 1826. Systematische Beschreibung der bekannten europäischen zweiflügeligen Insecten. Fünfter Theil. [5]. Hamm. $412 \mathrm{pp}$.

Merriam Webster. 1997. Merriam Webster's Geographical Dictionary. Third Edition, Springfield, MA, $1361 \mathrm{pp}$.

Ozerov A.L. 2009. New species of Scathophagidae (Diptera) // Russian Entomological Journal. Vol.17 (for 2008). No.4. P.419427.

Ozerov A.L., Barkalov A.V. 2014. On the fauna of Scathophagidae (Diptera) of Taimyr Peninsular (Russia: Krasnoyarskiy Kray) // Euroasian Entomological Journal. Vol.13. No.6. P.560-565.

Ozerov A.L., Krivosheina M.G. 2013b. New and little known palaearctic species of Scathophagidae (Diptera) // Far Eastern Entomologist. No.270. P.1-6.
Ozerov A.L., Krivosheina M.G. 2014. To the fauna of dung flies (Diptera: Scathophagidae) of Russian Far East // Russian Entomological Journal. Vol.23. No.3. P.203-222.

Ringdahl O. 1928. Neue skandinavische Dipteren // Entomologisk Tidskrift. Vol.49. P.18-21.

Šifner F. 2008. A catalogue of the Scathophagidae (Diptera) of the Palaearctic region, with notes on their taxonomy and faunistics // Acta Entomologica Musei Nationalis Pragae. Vol.48. No.1. P.111-196.

Šifner F. 2011. Two new species of Scathophagidae (Insecta: Diptera) from the Czech Republic, with description of the males of Scathophaga bohemiae and Norellisoma vonickai // Journal of the National Museum (Prague), Natural History Series. Vol.180. No.3. P.31-38.

Šifner F. 2013. Three new species of the family Scathophagidae (Diptera) from the Czech Republic and Slovakia // Journal of the National Museum (Prague), Natural History Series. Vol.182. No.3. P.35-50.

Stackelberg A.A. 1952. New species of Cordyluridae (Diptera) from North of USSR // Proceedings of the Zoological Institute of the USSR. Vol.12. P.405-407 [in Russian].

Stuckenberg B.R. 1999. Antennal evolution in the Brachycera (Diptera), with a reassessment of terminology relating to the flagellum // Studia Dipterologica. Vol.6. S.33-48.

Vockeroth J.R. 1965. Subfamily Scatophaginae // Stone A. et al. (eds.). A catalog of the Diptera of America north of Mexico. United States Department of Agriculture, Agriculture Handbook. No.276. P.826-842.

Vockeroth J.R. 1987. 103. Scathophagidae // McAlpine J.F. et al. (eds.). Manual of Nearctic Diptera. 2. Agriculture Canada, Research Branch, Ottawa. Monograph 28. P.1085-1097.

Zetterstedt J.W. [1838]. Sectio tertia. Diptera. P. 477-868 in Insecta Lapponica.'1840', Leopold Voss, Lipsiae [= Leipzig], vi + $1140 \mathrm{pp}$.

Zetterstedt J.W. 1846. Diptera Scandinaviae disposita et descripta. Tomus quintus. Ex officina lundbergiana, Lundae [= Lund]. P.1739-2162. 